\title{
TWO-LEVEL-SPLIT DECOMPOSITION OF ANISOTROPIC BESOV SPACES
}

\author{
WOLFGANG DAHMEN, SHAI DEKEL, AND PENCHO PETRUSHEV
}

\begin{abstract}
Anisotropic Besov spaces (B-spaces) are developed based on anisotropic multilevel ellipsoid covers (dilations) of $\mathbb{R}^{n}$. This extends earlier results on anisotropic Besov spaces. Furthermore, sequences of anisotropic bases are constructed and utilized for two-level-split decompositions of the B-spaces and nonlinear $m$-term approximation.
\end{abstract}

AMS Subject Classification: 46E35, 41A46, 41A25, 41A17

Key Words: Multilevel ellipsoid covers, homogeneous spaces, anisotropic Besov spaces, two-level-split bases, nonlinear approximation

\section{INTRODUCTION}

Anisotropic phenomena appear in various contexts in mathematical analysis and its applications. The formation of shocks results in jump discontinuities of solutions of hyperbolic conservation laws across lower dimanional manifolds and sharp edges often separate areas of little detail in digital images, to name just two examples. In the first case such jumps cause a serious obstruction to appropriate regularity theorems since the available regularity scales are either inherently isotropic or coordinate biased or are subject to an uncontrollable restricted regularity range, as will be pointed out below. In the second case the anisotropy makes it more difficult to come up with efficient encoding strategies that are entropy optimal when using cartoons as model classes. The central objective of this paper is to set up a sufficiently flexible framework for anisotropic regularity notions that at least bear a potential for dealing with questions of the above type.

Let us briefly relate now the present approach to earlier considerable efforts in extending the theory of classical Besov and Triebel-Lizorkin spaces to non-isotropic settings. One line of extension is concerned with function and distribution spaces on $\mathbb{R}^{n}$ furnished with anisotropic dilation structures. Various authors have considered dilations of $\mathbb{R}^{n}$ induced by a one parameter family of diagonal matrices of the form $\operatorname{diag}\left(2^{b_{1} t}, \ldots, 2^{b_{n} t}\right), b_{j}>0$. Calderón and Torchinsky $[4,5]$ have developed the so called parabolic Hardy spaces induces by certain one parameter dilation groups, which were further extended by Folland and Stein [12]. Bownik [1, 2] and Bownik and Ho [3] have considered dilations on $\mathbb{R}^{n}$ induced by the powers $A^{j}$ of

Date: January 18, 2007.

1991 Mathematics Subject Classification. Primary 46E35; Secodary 41A15, 41A25, 41A46.

Key words and phrases. Anisotropic Besov spaces, multilevel expansions, nonlinear approximation.

Address for manuscript correspondence: Pencho Petrushev, Department of Mathematics, University of South Carolina, Columbia, SC 29208, E-mail: pencho@math.sc.edu, Phone: (803)7776686, Fax: (803)777-6527 . 
an expansive matrix $A$ with eigenvalues $\lambda$ satisfying $|\lambda|>1$, and applied them to the development of anisotropic Besov and Triebel-Lizorkin spaces.

Yet another line of generalization of the classical spaces originated from the work of Coifman and Weiss $[6,7]$, where $\mathbb{R}^{n}$ was replaced by the more general spaces of homogeneous type. Spaces on homogeneous spaces have been further developed by several authors, in particular, more recently by Y. Han and his coauthors, see e.g. [13].

In this article we introduce and explore a new more general setting for anisotropic Besov spaces (homogeneous and inhomogeneous) on $\mathbb{R}^{n}$ of any positive smoothness that allows a lot more flexibility in dealing with anisotropic features of functions e.g. singularities along curves and lower dimensional smooth manifolds. Our approach is based on multilevel discrete (or continuous) anisotropic dilations of $\mathbb{R}^{n}$, where only mild conditions are imposed on the dilation matrices which still allow them to rapidly change from point to point and in depth, from level to level. These dilations are much more general than the dilations mentioned above which are shift invariant and where the different levels are obtained from powers of the same matrix or from a one parameter family of matrices. Instead of "dilations" we shall use the more convenient geometric term "ellipsoid cover of $\mathbb{R}^{n}$ ".

As will be shown, $\mathbb{R}^{n}$ equipped with a discrete or continuous ellipsoid cover becomes a homogeneous space and therefore the general theory of Besov spaces on homogeneous spaces applies. However, this theory only allows one to handle Besov and Triebel-Lizorkin spaces of smoothness $\alpha$ with $|\alpha|<\varepsilon$, where $\varepsilon>0$ is sufficiently small.

Although very general our setup allows us to overcome this latter principal limitation. For a given discrete multilevel ellipsoid cover $\Theta$ of $\mathbb{R}^{n}$ (see $\S 2.1$ ), we define the B-spaces $\dot{B}_{p q}^{\alpha}(\Theta)$ and $B_{p q}^{\alpha}(\Theta)$ via local polynomial approximation on all ellipsoids from $\Theta$. This approach enables us indeed to develop the anisotropic Besov spaces (B-spaces) $\dot{B}_{p q}^{\alpha}$ and $B_{p q}^{\alpha}$ for arbitrarily large $\alpha>0$ which is the principle difference between our theory and the general theory of Besov spaces on homogeneous spaces. Our B-spaces can be viewed as a generalization of the classical Besov spaces as well as the anisotropic Besov spaces studied by Bownik [1] with weight 1 (see $\S 5.3$ ). For the theory of classical Besov spaces we refer the reader to $[16,18,19]$.

The highly anisotropic locally and scalewise varying structure of the dilations considered here prevents us from using Fourier analysis techniques; we use piecewise polynomials instead. A key ingredient in our theory is a certain two-level-split decomposition of B-spaces. For any given anisotropic cover $\Theta$ we construct a multilevel sequence of bases which provide a means for decomposing the associated B-spaces. The basis elements are compactly supported piecewise polynomials which are well aligned with the anisotropic space dilations induced by $\Theta$. However, since they do not oscillate sufficiently, they are directly applicable only to B-spaces of positive smoothness. The two-level-split decomposition of functions originates from [9], where it is applied in a regular setup and used for the purpose of preconditioning systems of equations stemming from mesh free discretizations of elliptic boundary value problems.

Of course, the usefulness of any regularity notion relies on whether it actually conveys sufficient information about the intrinsic complexity of corresponding functions, e.g. expressed in terms of (quasi-)sparse representations with respect to a suitable system of building blocks. Just as one may expect we show that the 
B-spaces $\dot{B}_{\tau \tau}^{\alpha}(\Theta)$ with $1 / \tau=\alpha / n+1 / p$ govern the rates of nonlinear $m$-term $L_{p^{-}}$ approximation from the two-level-split bases induced by $\Theta$. This suggests to adapt $\Theta$ to the approximand. Accordingly, we would like to put forward here a new adaptive strategy for measuring the smoothness of functions, namely, by choosing from the collection of all anisotropic B-spaces, induced by an admissible set of ellipsoid covers of $\mathbb{R}^{n}$, one that maximizes the regularity index viz. rate of best $m$-term approximation .

To better illustrate our adaptive approach to smoothness we discuss two examples that are very simple but nevertheless reveal some perhaps unexpected effects. As for the first example, we show in $\S 7.1$ that for a suitable ellipsoid cover $\Theta$ the B-space smoothness of the characteristic function of the unit ball $B(0,1) \subset \mathbb{R}^{2}$ in $\dot{B}_{\tau \tau}^{\alpha}(\Theta)$ is essentially $4 / p$, while in the corresponding (classical isotropic) Besov spaces it is $2 / p$. More strikingly, in the adaptive B-space scales $\dot{B}_{\tau \tau}^{\alpha}(\Theta)$ the smoothness of the characteristic function of any square $Q \subset \mathbb{R}^{2}$ is arbitrarily high, i.e. can be any $\alpha>0$ while in the corresponding isotropic Besov spaces it is essentially $2 / p$ (see §7.2). However, it is important to note that the cover $\Theta$ needed to describe that level of smoothness depends on $\alpha$, a fact that has some interesting consequences worth a brief discussion (see $\S 7.3$ ). As a consequence, the rates of adaptive $m$-term approximation for these model functions from the two-level-split bases are better than e.g. the rates of $m$-term approximation from wavelets.

Let us finally mention that more special anisotropic B-spaces of technically different nature but somewhat similar in spirit have been introduced and used in $[14,10]$ (see also $[8,15,17]$ ). These are B-spaces generated by multilevel nested triangulations and are defined via local piecewise polynomial approximation. They are specifically designed for the purpose of nonlinear $m$-term spline approximation, and consequently are much more specialized than the B-spaces of this paper. Moreover, it is important to note that insisting on hierarchies of nested partitions entails a distinct lack of flexibility compared to the present approach since, for instance, the successors of a triangle with a sharp angle will always exhibit angles at least that sharp. Yet another type of anisotropic Besov spaces has been considered in [11].

The outline of the paper is the following. In $\S 2$ we introduce and give some basic properties of the continuous and discrete multilevel covers of $\mathbb{R}^{n}$. In $\S 3$ we construct the bases induced by discrete multilevel covers of $\mathbb{R}^{n}$ and establish their stability. In $\S 4$ we utilize the bases from $\S 3$ for the construction of two-level-split bases and decompositions. In $\S 5$ we define the homogeneous and inhomogeneous B-spaces induced by multilevel covers of $\mathbb{R}^{n}$ and establish the equivalence of various B-norms. Section 6 is devoted to nonlinear $m$-term approximation from two-level-split bases. In $\S 7$ we show that the characteristic functions of the unit ball and any square in $\mathbb{R}^{n}$ have higher adaptive B-space smoothness than their regular Besov space smoothness. Section 8 is an appendix where we give the proof of the equivalence of several B-space norms.

Throughout the paper we use the following notation: $\|f\|_{p}:=\|f\|_{L_{p}\left(\mathbb{R}^{n}\right)}$; for a measurable set $E \subset \mathbb{R}^{n},|E|$ denotes the Lebesgue measure of $E, \mathbb{1}_{E}$ is the characteristic function of $E ; \mathcal{P}_{k}$ denotes the set of all algebraic polynomials in $n$ variables of degree $\leq k-1$. The Schwartz class of rapidly decaying $C^{\infty}$ functions on $\mathbb{R}^{n}$ is denoted by $\mathcal{S}$ and its dual by $\mathcal{S}^{\prime}$. Positive constants are denoted by 
$c, c_{1}, c_{*}, \ldots$ and they may vary at every occurrence; $A \sim B$ means $c_{1} A \leq B \leq c_{2} A$ for some unspecified but fixed constants $c_{1}, c_{2}$.

\section{Anisotropic Multilevel Ellipsoid Covers of $\mathbb{R}^{n}$}

We begin with some preliminaries. The image of the unit ball $B^{*}:=B(0,1)$ in $\mathbb{R}^{n}$ via an affine transform will be called an ellipsoid. For a given ellipsoid $\theta \subset \mathbb{R}^{n}$, we let $A_{\theta}$ be an affine transform such that $\theta=A_{\theta}\left(B^{*}\right)$. Denoting by $v_{\theta}:=A_{\theta}(0)$ the "center" of $\theta$, we have

$$
A_{\theta}(x)=M_{\theta} x+v_{\theta}
$$

where $M_{\theta}$ is a nonsingular $n \times n$ matrix.

It will be convenient for us to use the singular value decomposition of matrices, that is, any nonsingular real matrix $M \in \mathbb{R}^{n \times n}$ can be represented in the form $M=U D V$, where the matrices $U$ and $V$ are $n \times n$ orthogonal matrices and $D$ is diagonal and $D=\operatorname{diag}\left(\sigma_{1}, \sigma_{2}, \ldots, \sigma_{n}\right)$ with $\sigma_{1} \geq \sigma_{2} \geq \cdots \geq \sigma_{n}>0$. It is easy to see that $\sigma_{1}^{2} \geq \cdots \geq \sigma_{n}^{2}$ are the eigenvalues of $M^{T} M$ and $\sigma_{1}^{-2} \leq \cdots \leq \sigma_{n}^{-2}$ are the eigenvalues of $\left(M^{-1}\right)^{T} M^{-1}$. Hence

$$
\|M\|_{\ell_{2} \rightarrow \ell_{2}}=\sigma_{1} \quad \text { and } \quad\left\|M^{-1}\right\|_{\ell_{2} \rightarrow \ell_{2}}=1 / \sigma_{n} .
$$

These norms have a clear geometric meaning. Thus if $M_{\theta}$ is as in (2.1), then $\operatorname{diam} \theta=2\left\|M_{\theta}\right\|_{\ell_{2} \rightarrow \ell_{2}}$.

2.1. Discrete ellipsoid covers (dilations) of $\mathbb{R}^{n}$. The notion of a discrete multilevel ellipsoid cover of $\mathbb{R}^{n}$ we introduce and explore here will play a central role in this study.

Definition 2.1. We call

$$
\Theta=\bigcup_{m \in \mathbb{Z}} \Theta_{m}
$$

$a$ discrete multilevel ellipsoid cover of $\mathbb{R}^{n}$ if the following conditions are obeyed, where $a_{0}, a_{1}, \ldots, a_{8}$, and $N_{1}$ are positive constants:

(a) Every level $\Theta_{m}(m \in \mathbb{Z})$ consists of ellipsoids $\theta$ such that

$$
a_{1} 2^{-a_{0} m} \leq|\theta| \leq a_{2} 2^{-a_{0} m}
$$

and $\Theta_{m}$ is a cover of $\mathbb{R}^{n}$, i.e. $\mathbb{R}^{n}=\bigcup_{\theta \in \Theta_{m}} \theta$.

(b) For each $\theta \in \Theta$ let $A_{\theta}$ be an affine transform, associated with $\theta$, of the form

$$
A_{\theta}(x)=M_{\theta} x+v_{\theta}, \quad M_{\theta} \in \mathbb{R}^{n \times n},
$$

such that $\theta=A_{\theta}\left(B^{*}\right)$ and $v_{\theta}:=A(0)$ is the center of $\theta$. We postulate that for any $\theta \in \Theta_{m}(m \in \mathbb{Z})$ and $\theta^{\prime} \in \Theta_{m+\nu}(\nu \geq 0)$ with $\theta \cap \theta^{\prime} \neq \emptyset$, we have

$$
a_{3} 2^{-a_{4} \nu} \leq 1 /\left\|M_{\theta^{\prime}}^{-1} M_{\theta}\right\|_{\ell_{2} \rightarrow \ell_{2}} \leq\left\|M_{\theta}^{-1} M_{\theta^{\prime}}\right\|_{\ell_{2} \rightarrow \ell_{2}} \leq a_{5} 2^{-a_{6} \nu} .
$$

(c) At most $N_{1}$ ellipsoids from $\Theta_{m}$ can intersect at a time.

(d) For every $x \in \mathbb{R}^{n}$ and $m \in \mathbb{Z}$ there exists $\theta \in \Theta_{m}$ such that $x \in \theta^{\diamond}$, where $\theta^{\diamond}$ is the dilated version of $\theta$ by a factor of $a_{7}<1$, i.e. $\theta^{\diamond}=A_{\theta}\left(B\left(0, a_{7}\right)\right)$.

(e) If $\theta \cap \eta \neq \emptyset$ with $\theta \in \Theta_{m}$ and $\eta \in \Theta_{m} \cup \Theta_{m+1}$, then $|\theta \cap \eta|>a_{8}|\eta|$. 
Remarks: 1. Any discrete multilevel ellipsoid cover $\Theta$ of $\mathbb{R}^{n}$ depends on a collection of parameters (which are not necessarily independent) that contain all constants appearing in conditions (a)-(e) of Definition 2.1. We shall denote this set of parameters by $\mathbf{p}(\Theta)$.

2. It is important to note that the set of all ellipsoid covers of $\mathbb{R}^{n}$ is invariant under affine transforms. More precisely, the images $A(\theta)$ of all ellipsoids $\theta \in \Theta$ of a given cover $\Theta$ of $\mathbb{R}^{n}$ via an affine transform $A$ of the form $A(x)=M x+v$ with $|\operatorname{det} M|=1$ form an ellipsoid cover of $\mathbb{R}^{n}$ with the same parameters as the parameters of $\Theta$. If $|\operatorname{det} M| \neq 1$ then only the constants $a_{1}$ and $a_{2}$ in condition (a) should be changed accordingly.

3. Condition (b) indicates that if $\theta \cap \theta^{\prime} \neq \emptyset$, then the ellipsoids $\theta$ and $\theta^{\prime}$ cannot change uncontrollably in shape and orientation when they are from close levels. More precisely, denote $M:=M_{\theta}^{-1} M_{\theta^{\prime}}$ and let $M=U D V$ be the singular value decomposition of $M$, where $U$ and $V$ are orthogonal matrices, and $D=\operatorname{diag}\left(\sigma_{1}, \sigma_{2}, \ldots, \sigma_{n}\right)$ is diagonal with $\sigma_{1} \geq \sigma_{2} \geq \cdots \geq \sigma_{n}>0$. As in (2.2)

$$
\|M\|_{\ell_{2} \rightarrow \ell_{2}}=\sigma_{1} \quad \text { and } \quad\left\|M_{\theta^{\prime}}^{-1} M_{\theta}\right\|_{\ell_{2} \rightarrow \ell_{2}}=\left\|M^{-1}\right\|_{\ell_{2} \rightarrow \ell_{2}}=1 / \sigma_{n} .
$$

Therefore, condition (b) is equivalently expressed as

$$
a_{3} 2^{-a_{4} \nu} \leq \sigma_{n} \leq \cdots \leq \sigma_{1} \leq a_{5} 2^{-a_{6} \nu} .
$$

This condition also has a clear geometric interpretation: The affine transform $A_{\theta}^{-1}$, which maps the ellipsoid $\theta$ onto the unit ball $B^{*}$, maps the ellipsoid $\theta^{\prime}$ onto an ellipsoid with semi-axes $\sigma_{1}, \sigma_{2}, \ldots, \sigma_{n}$ satisfying (2.4).

4. Evidently the sign of $\nu$ in condition (b) can be reversed. Namely, condition (b) is equivalent to the following condition.

$\left(b^{\prime}\right)$ If $\theta \in \Theta_{m}$ and $\theta^{\prime} \in \Theta_{m-\nu}(\nu \geq 0)$, with $\theta \cap \theta^{\prime} \neq \emptyset$, then

$$
\left(1 / a_{5}\right) 2^{a_{6} \nu} \leq 1 /\left\|M_{\theta^{\prime}}^{-1} M_{\theta}\right\|_{\ell_{2} \rightarrow \ell_{2}} \leq\left\|M_{\theta}^{-1} M_{\theta^{\prime}}\right\|_{\ell_{2} \rightarrow \ell_{2}} \leq\left(1 / a_{3}\right) 2^{a_{4} \nu} .
$$

Therefore, if $M:=M_{\theta}^{-1} M_{\theta^{\prime}}=U D V$ with $D=\operatorname{diag}\left(\sigma_{1}, \sigma_{2}, \ldots, \sigma_{n}\right)$ as above, then (2.5) is equivalent to

$$
\left(1 / a_{5}\right) 2^{a_{6} \nu} \leq \sigma_{n} \leq \cdots \leq \sigma_{1} \leq\left(1 / a_{3}\right) 2^{a_{4} \nu}
$$

5 . We need to interrelate the semi-axes of intersecting ellipsoids from $\Theta$. For a given $\theta \in \Theta$ denote

$$
\sigma_{\max }(\theta):=\left\|M_{\theta}\right\|_{\ell_{2} \rightarrow \ell_{2}} \quad \text { and } \quad \sigma_{\min }(\theta):=\left\|M_{\theta}^{-1}\right\|_{\ell_{2} \rightarrow \ell_{2}}^{-1} .
$$

These are the maximum and minimum semi-axes of the ellipsoid $\theta$.

Lemma 2.2. If $\theta \in \Theta_{m}, \theta^{\prime} \in \Theta_{m+\nu}, \nu \geq 0$, and $\theta \cap \theta^{\prime} \neq \emptyset$, then

$$
a_{3} 2^{-a_{4} \nu} \sigma_{\max }(\theta) \leq \sigma_{\max }\left(\theta^{\prime}\right) \leq a_{5} 2^{-a_{6} \nu} \sigma_{\max }(\theta)
$$

and

$$
a_{3} 2^{-a_{4} \nu} \sigma_{\min }(\theta) \leq \sigma_{\min }\left(\theta^{\prime}\right) \leq a_{5} 2^{-a_{6} \nu} \sigma_{\min }(\theta) .
$$

Proof. Let us abbreviate $\|\cdot\|:=\|\cdot\|_{\ell_{2} \rightarrow \ell_{2}}$. By property $(b)$ of $\Theta$ we have

$$
\left\|M_{\theta^{\prime}}\right\| \leq\left\|M_{\theta}\right\| \cdot\left\|M_{\theta}^{-1} M_{\theta^{\prime}}\right\| \leq a_{5} 2^{-a_{6} \nu}\left\|M_{\theta}\right\|
$$

and by $\left(b^{\prime}\right)$

$$
\left\|M_{\theta}\right\| \leq\left\|M_{\theta}^{\prime}\right\| \cdot\left\|M_{\theta^{\prime}}^{-1} M_{\theta}\right\| \leq\left(1 / a_{3}\right) 2^{a_{4} \nu}\left\|M_{\theta^{\prime}}\right\|,
$$

which yield (2.8). One similarly proves (2.9). 
6. Condition (b) can be replaced by the following slightly stronger but more convenient condition:

$\left(b^{\prime \prime}\right)$ For any $\theta, \theta^{\prime} \in \Theta_{m}(m \in \mathbb{Z})$ with $\theta \cap \theta^{\prime} \neq \emptyset$

$$
a_{3} \leq 1 /\left\|M_{\theta^{\prime}}^{-1} M_{\theta}\right\|_{\ell_{2} \rightarrow \ell_{2}} \leq\left\|M_{\theta}^{-1} M_{\theta^{\prime}}\right\|_{\ell_{2} \rightarrow \ell_{2}} \leq a_{5}, \quad a_{3} \leq 1 \leq a_{5} .
$$

and for any $\theta \in \Theta_{m}$ and $\theta^{\prime} \in \Theta_{m+1}(m \in \mathbb{Z})$ with $\theta \cap \theta^{\prime} \neq \emptyset$

$$
2^{-a_{4}} \leq 1 /\left\|M_{\theta^{\prime}}^{-1} M_{\theta}\right\|_{\ell_{2} \rightarrow \ell_{2}} \leq\left\|M_{\theta}^{-1} M_{\theta^{\prime}}\right\|_{\ell_{2} \rightarrow \ell_{2}} \leq 2^{-a_{6}} .
$$

It is readily seen that this condition implies condition (b).

7. By property $\left(b^{\prime}\right)$ (which is equivalent to (b)) it follows that the ellipsoids of $\Theta_{m}$ expand to $\mathbb{R}^{n}$ as $m \rightarrow-\infty$; more precisely if $\left(\theta_{m}\right)_{m \leq 0}$ is a sequence of ellipsoids $\theta_{m} \in \Theta_{m}$ which contain a fixed point $x \in \mathbb{R}^{n}$, then $\cup_{m \leq 0} \theta_{m}=\mathbb{R}^{n}$. In the other direction, property (b) implies that for any compact $K \subset \mathbb{R}^{n}$

$$
\max \left\{\operatorname{diam} \theta: \theta \in \Theta_{m}, \theta \cap K \neq \emptyset\right\} \rightarrow 0 \quad \text { as } m \rightarrow \infty .
$$

8. Property (d) indicates that every point $x \in \mathbb{R}^{n}$ is "well covered" by at least one ellipsoid from every level $\Theta_{m}$.

9. Condition (e) may seem restrictive, but the next observation shows that this is not the case.

Lemma 2.3. Suppose $\Theta$ is a discrete multilevel ellipsoid cover of $\mathbb{R}^{n}$ satisfying conditions $(a)-(d)$ above. Then there exists a discrete multilevel ellipsoid cover $\widetilde{\Theta}$ of $\mathbb{R}^{n}$ with properties $(a)-(e)$ (with possibly different constants $a_{1}$ and $a_{7}$ ) obtained by dilating every ellipsoid $\theta \in \Theta$ by a factor $r_{\theta}$ satisfying $\left(a_{7}+1\right) / 2 \leq r_{\theta} \leq 1$.

Proof. The idea is that slight dilations of each $\theta \in \Theta$, one at a time, allows one to realize in addition (e). In fact, evidently, there exist constants $N_{0}$ and $N_{2}$ depending on the parameters of $\Theta$ such that each ellipsoid $\theta \in \Theta_{m}(m \in \mathbb{Z})$ can be intersected by at most $N_{2}$ ellipsoids from $\Theta_{m+1}$ and by at most $N_{0}$ ellipsoids from $\Theta_{m-1}$. Now set $N:=2\left(N_{0}+N_{1}+N_{2}\right)+1, b:=\left(a_{7}+1\right) / 2$, and $\delta:=(1-b) / N$.

For a fixed $\theta \in \Theta_{m}$, denote $\theta_{j}:=A_{\theta}(B(0, b+j \delta)), j=0,1, \ldots, N$. So, $\theta_{j}$ is the dilated version of $\theta$ by a factor of $b+j(1-b) / N=\left(1-\frac{j}{N}\right) b+\frac{j}{N}$. Now, let $S_{j}:=\theta_{j} \backslash \theta_{j-1}, j=1,2, \ldots, N$, be the shells induced by the ellipsoids $\theta_{j}$. Also, set $S_{0}:=\theta_{0}$ and $S_{N+1}:=\mathbb{R}^{n} \backslash \theta$. We shall call a shell $S_{j}(1 \leq j \leq N)$ a "bad shell" if there is an ellipsoid $\eta \in \Theta_{m-1} \cup \Theta_{m} \cup \Theta_{m+1}, \eta \neq \theta$, such that $\eta \cap S_{j} \neq \emptyset$ and either $\eta \cap S_{j-1}=\emptyset$ or $\eta \cap S_{j+1}=\emptyset$. It is readily seen that a single ellipsoid $\eta$ can spoil at most two shells $S_{j}$ and hence at most $2\left(N_{0}+N_{1}+N_{2}\right)$ shells can be spoiled by ellipsoids from $\Theta_{m-1} \cup \Theta_{m} \cup \Theta_{m+1}$. Since there are $N=2\left(N_{0}+N_{1}+N_{2}\right)+1$ shells at least one shell, say, $S_{j_{0}}\left(1 \leq j_{0} \leq N\right)$ will be "good". Then it is easy to see that if $\eta \cap \theta_{j_{0}} \neq \emptyset$ for some $\eta \in \Theta_{m-1} \cup \Theta_{m} \cup \Theta_{m+1}$, then $\left|\theta_{j_{0}} \cap \eta\right| \geq a_{8}|\eta|$ for some constant $a_{8}>0$ depending only on the parameters of $\Theta$. Thus the dilated version $\theta_{j_{0}}$ of $\theta$ has the desired property.

One completes the proof of this proposition inductively by processing as above all ellipsoids from $\Theta$ ordered in a sequence. The rule is that once an ellipsoid from $\Theta$ has been processed it will never be touched again.

Examples: A first class of examples is based on the so called Strong Locally Regular (SLR) multilevel triangulations that have been introduced in [14]. They provide another means for constructing discrete ellipsoid covers of $\mathbb{R}^{2}$. Given an SLR-triangulation $\mathcal{T}$, one considers for each triangle $\triangle \in \mathcal{T}$ the minimum area 
circumscribed ellipse. Then one dilates the resulting ellipses by a sufficiently large factor $>1$ to obtain a discrete ellipse cover of $\mathbb{R}^{2}$. The main advantage of ellipse covers over SLR-triangulations is that the latter are nested which makes them less flexible and harder to construct.

It will be shown in Lemma 2.9, how to derive discrete multilevel covers with the above properties from so called continuous or semi-continuous ellipsoid covers that will be introduced next and are easier to construct. Any example of such a cover therefore provides an example for the discrete setting as well.

2.2. Continuous covers and quasi-distance. As mentioned before, the essential developments of this paper are based on the above discrete multilevel covers. Nevertheless, we pause to introduce next two variants, namely continuous and semicontinuous covers, for the following reasons. First, while on the one hand these variants are easier to construct, as reflected by several examples given below, we shall give, on the other hand, a general mechanism that generates from any continuous cover a discrete one satisfying properties (a) - (e). In this sense these relaxed notions simplify the realization of concrete examples of discrete covers. Second, they will later be used for the regularity analysis of the examples in $\S 7$. Moreover, we shall see that (all versions of) covers give rise to quasi-distances which allows us to relate the present results to previously known results concerning Besov spaces on homogeneous spaces.

Definition 2.4. We say that

$$
\Theta:=\bigcup_{t \in \mathbb{R}} \Theta_{t}
$$

is a continuous multilevel ellipsoid cover of $\mathbb{R}^{n}$ or a continuous system of dilations of $\mathbb{R}^{n}$ if it satisfies the following conditions, where $a_{0}, \ldots, a_{5}$ are positive constants:

(a) For every $v \in \mathbb{R}^{n}$ and $t \in \mathbb{R}$ there exists an ellipsoid $\theta(v, t) \in \Theta_{t}$ and an affine transform $A_{v, t}$ such that

$$
|\theta(v, t)|=a_{1} 2^{-a_{0} t}
$$

$\theta(v, t)=A_{v, t}\left(B^{*}\right)$, and $A_{v, t}$ is of the form $A_{v, t}(x)=M_{v, t} x+v$, where $M_{v, t}$ is a nonsingular $n \times n$ matrix.

(b) For any $v, y \in \mathbb{R}^{n}, t \in \mathbb{R}$, and $s>0$, if $\theta(v, t) \cap \theta(y, t+s) \neq \emptyset$, then

$$
a_{2} 2^{-a_{3} s} \leq 1 /\left\|M_{y, t+s}^{-1} M_{v, t}\right\|_{\ell_{2} \rightarrow \ell_{2}} \leq\left\|M_{v, t}^{-1} M_{y, t+s}\right\|_{\ell_{2} \rightarrow \ell_{2}} \leq a_{4} 2^{-a_{5} s} .
$$

For continuous covers also the scale is allowed to vary continuously. The following variant takes us half way towards the fully discrete setting introduced in the previous setting.

Definition 2.5. We call

$$
\Theta=\bigcup_{m \in \mathbb{Z}} \Theta_{m}
$$

a multilevel semi-continuous ellipsoid cover of $\mathbb{R}^{n}$ if the following conditions are obeyed, where $a_{0}, \ldots, a_{6}$ are positive constants:

(a) For every $v \in \mathbb{R}^{n}$ and $m \in \mathbb{Z}$ there exists an ellipsoid $\theta(v, m) \in \Theta_{m}$ and an affine transform $A_{v, m}$ such that

$$
a_{1} 2^{-a_{0} m} \leq|\theta(v, m)| \leq a_{2} 2^{-a_{0} m},
$$

$\theta(v, m)=A_{v, m}\left(B^{*}\right)$, and $A_{v, m}$ is of the form $A_{v, m}(x)=M_{v, m} x+v$, where $M_{v, m}$ is a nonsingular $n \times n$ matrix. 
(b) For any $v, y \in \mathbb{R}^{n}, m \in \mathbb{Z}$, and $\nu>0$, if $\theta(v, m) \cap \theta(y, m+\nu) \neq \emptyset$, then

$$
a_{3} 2^{-a_{4} s \nu} \leq 1 /\left\|M_{y, m+\nu}^{-1} M_{v, m}\right\|_{\ell_{2} \rightarrow \ell_{2}} \leq\left\|M_{v, m}^{-1} M_{y, m+\nu}\right\|_{\ell_{2} \rightarrow \ell_{2}} \leq a_{5} 2^{-a_{6} \nu} .
$$

It is readily see that any continuous ellipsoid cover $\Theta$ of $\mathbb{R}^{n}$ induces a semicontinuous ellipsoid cover by defining $\Theta_{m}$ as $\Theta_{t}$ with $t=m$.

Examples. (i) The regular cover of $\mathbb{R}^{n}$ consisting of all balls in $\mathbb{R}^{n}$ is the simplest example of a continuous ellipsoid (ball) cover of $\mathbb{R}^{n}$.

(ii) The one parameter family of diagonal dilation matrices

$$
D_{t}=\operatorname{diag}\left(2^{-t b_{1}}, 2^{-t b_{2}}, \ldots, 2^{-t b_{n}}\right), \quad b_{j}>0, j=1, \ldots, n,
$$

apparently induces a continuous ellipsoid cover of $\mathbb{R}^{n}$.

(iii) In $[4,5]$ Calderón and Torchinsky developed the so called parabolic Hardy spaces generated by continuous dilation matrices associated with a continuous semigroup $A_{t}, t>0, A_{s t}:=A_{t} A_{s}$ of affine transforms on $\mathbb{R}^{n}$ leaving the origin fixed and satisfying

$$
t^{\alpha} \leq\left\|A_{t}\right\|_{\ell_{2} \rightarrow \ell_{2}} \leq t^{\beta}, \quad t \geq 1
$$

where $1 \leq \alpha \leq \beta<\infty$. One can easily see that any such semigroup of matrices gives rise to a continuous ellipsoid cover (dilation) of $\mathbb{R}^{n}$.

(iv) Consider an arbitrary $n \times n$ real matrix $A$ with eigenvalues $\lambda$ satisfying $|\lambda|>1$. Then it is easy to see that the affine transforms $A_{v, m}(x):=A^{-m} x+v$, $v \in \mathbb{R}^{n}, m \in \mathbb{Z}$, define a semi-continuous ellipsoid cover (dilations) in the sense of Definition 2.5. This particular kind of dilations are used in $[1,2,3]$ for the development of anisotropic Hardy, Besov, and Triebel-Lizorkin spaces.

(v) In Section 7, we construct two nontrivial continuous covers of $\mathbb{R}^{2}$. These are examples of anisotropic ellipsoid covers where the ellipsoids change rapidly from point to point and in depth.

In the following we first show that a quasi-distance is naturally associated with any continuous or semi-continuous or discrete ellipsoid covers of $\mathbb{R}^{n}$. Second, we prove that $\mathbb{R}^{n}$ equipped with a distance of this sort and the Lebesgue measure becomes a space of homogeneous type. Thus the anisotropic Besov spaces we develop in this paper are in particular spaces on homogeneous type spaces.

We begin by recalling the definition of a quasi-distance on a set $X \neq \emptyset$ : A map $\rho: X \times X \rightarrow[0, \infty)$ is called a quasi-distance on $X$ if for any $x, y, z \in X$

(i) $\rho(x, y)=0 \Longleftrightarrow x=y$,

(ii) $\rho(y, x)=\rho(x, y)$,

(iii) $\rho(x, z) \leq K(\rho(x, y)+\rho(y, z))$ with $K \geq 1$.

Definition 2.6. Assuming that $\Theta$ is a continuous or semi-continuous or discrete ellipsoid cover of $\mathbb{R}^{n}$, we define

$$
\rho(x, y):=\min \{|\theta|: \theta \in \Theta \text { and } x, y \in \theta\}, \quad x, y \in \mathbb{R}^{n} .
$$

Note that in order to cover the standard Euclidean distance we should take the $n$th root of $\rho(x, y)$ defined above. But since this would only change the constant $K$ in (iii) while adding to technicalities we prefer to work with the above version.

Proposition 2.7. For any continuous, semi-continuous or discrete cover $\Theta$ of $\mathbb{R}^{n}$ the map $\rho: \mathbb{R}^{n} \times \mathbb{R}^{n} \rightarrow[0, \infty)$ defined above is a quasi-distance on $\mathbb{R}^{n}$. 
We shall prove this proposition only in the case when $\Theta$ is a discrete cover of $\mathbb{R}^{n}$. The proof when $\Theta$ is a continuous or semi-continuous cover is similar or even easier. The claim will follow from the following lemma.

Lemma 2.8. There is an integer $s_{*} \geq 1$ depending only on $\mathbf{p}(\Theta)$ such that for any ellipsoids $\theta \in \Theta_{m}(m \in \mathbb{Z})$ and $\theta^{\prime} \in \bar{\Theta}_{m+\nu}(\nu \geq 0)$ with $\theta \cap \theta^{\prime} \neq \emptyset$ and any $j \geq s_{*}$ there exists an ellipsoid $\eta_{j} \in \Theta_{m-j}$ such that $\theta \cup \theta^{\prime} \subset \eta_{j}$.

Proof. Let $\omega^{\prime}:=A_{\theta}^{-1}\left(\theta^{\prime}\right)$ and recall that $A_{\theta}^{-1}(\theta)=B^{*}$. By property (b) of $\Theta$ it follows that

$$
\operatorname{diam} \omega^{\prime}=2\left\|M_{\theta}^{-1} M_{\theta^{\prime}}\right\|_{\ell_{2} \rightarrow \ell_{2}} \leq 2 a_{5} 2^{-a_{0} \nu} \leq 2 a_{5}
$$

and hence

$$
A_{\theta}^{-1}\left(\theta \cup \theta^{\prime}\right)=B^{*} \cup \omega^{\prime} \subset B\left(0,1+2 a_{5}\right) .
$$

By property (d) of $\Theta$ for any $j \geq 1$ there exists $\theta_{j} \in \Theta_{m-j}$ such that $v_{\theta} \in$ $\theta_{j}^{\diamond}$, where $\theta_{j}^{\diamond}:=A_{\theta_{j}}\left(B\left(0, a_{7}\right)\right)$ is the dilated $\theta_{j}$ by a factor of $a_{7}<1$. Denote $\omega_{j}:=A_{\theta}^{-1}\left(\theta_{j}\right)$ and let $\omega_{j}^{\diamond}$ be the dilated $\omega_{j}$ by a factor of $a_{7}$. Also, denote by $\sigma_{1} \geq \sigma_{2} \geq \cdots \geq \sigma_{n}$ the semi-axes of the ellipsoid $\omega_{j}$. By property $\left(b^{\prime}\right)$ (see $(2.6)$ ) which is equivalent to property (b) it follows that

$$
\sigma_{n} \geq\left(1 / a_{5}\right) 2^{a_{6} j}
$$

On the other hand, by a simple geometric property of ellipsoids

$$
\operatorname{dist}\left(\omega_{j}^{\diamond}, \partial \omega_{j}\right)=\left(1-a_{7}\right) \sigma_{n}
$$

where $\operatorname{dist}\left(E_{1}, E_{2}\right)$ denotes the Euclidean distance between the sets $E_{1}, E_{2} \subset \mathbb{R}^{n}$. From this and (2.16) it follows that

$$
\operatorname{dist}\left(\omega_{j}^{\diamond}, \partial \omega_{j}\right) \geq \frac{1-a_{7}}{a_{5}} 2^{a_{6} j}
$$

Now, choose $s_{*} \geq 1$ so that

$$
\frac{1-a_{7}}{a_{5}} 2^{a_{6} s_{*}} \geq 1+2 a_{5} .
$$

Let $j \geq s_{*}$. Since $v_{\theta} \in \theta_{j}^{\diamond}$, then $0 \in \omega_{j}^{\diamond}$ and using (2.15), (2.17) and (2.18) we infer $A_{\theta}^{-1}\left(\theta \cup \theta^{\prime}\right) \subset \omega_{j}$ which implies $\theta \cup \theta^{\prime} \subset A_{\theta}\left(\omega_{j}\right)=: \eta_{j}$. This completes the proof.

Proof of Proposition 2.7. Remark 7 gives property (i). Property (ii) is obvious by the definition of $\rho(\cdot, \cdot)$. To show (iii), let $x, y, z \in \mathbb{R}^{n}$ and assume that $\rho(x, z)=$ $|\theta|, x, z \in \theta$, and $\rho(z, y)=\left|\theta^{\prime}\right|, z, y \in \theta^{\prime}$, where $\theta \in \Theta_{m}$ and $\theta^{\prime} \in \Theta_{m+\nu}$. Without loss of generality we may assume that $\nu \geq 0$. We now apply Lemma 2.8 to conclude that there exists an ellipsoid $\eta \in \Theta_{m-s_{*}}$ such that $\theta \cup \theta^{\prime} \subset \eta$ and hence

$$
\rho(x, y) \leq|\eta| \leq a_{2} 2^{-a_{0}\left(m-s_{*}\right)} \leq\left(a_{2} / a_{1}\right) 2^{a_{0} s_{*}}\left(|\theta|+\left|\theta^{\prime}\right|\right) \leq K(\rho(x, z)+\rho(z, x))
$$

The following observation justifies exploiting the greater comfort of continuous or semi-continuous covers.

Lemma 2.9. For every continuous or semi-continuous ellipsoid cover $\Theta$ of $\mathbb{R}^{n}$ there is a discrete ellipsoid cover $\widehat{\Theta}$ such that each ellipsoid $\theta \in \widehat{\Theta}$ is obtained from an ellipsoid in $\Theta$ by dilation by a factor $r_{\theta}$ obeying $\left(a_{7}+1\right) / 2 \leq r_{\theta} \leq 1$.

Furthermore, the quasi-distances $\rho(\cdot, \cdot)$ and $\hat{\rho}(\cdot, \cdot)$ induced by $\Theta$ and $\widehat{\Theta}$ are equivalent, i.e. $\rho(x, y) \sim \hat{\rho}(x, y)$ for $x, y \in \mathbb{R}^{n}$. 
Proof. We may assume that $\Theta$ is a semi-continuous ellipsoid cover of $\mathbb{R}^{n}$ for otherwise we construct one from the given continuous cover.

We first construct for every $m \in \mathbb{Z}$ a countable set $\widehat{\Theta}_{m} \subset \Theta_{m}$ satisfying conditions (c)-(d) of Definition 2.1. This can be done e.g. in two steps as follows: We first choose countably many ellipsoids from $\Theta_{m}$ so that condition (d) is fulfilled, and then inductively remove from this collection one-by-one all ellipsoids which do not destroy condition (d). After that condition (c) will be automatically fulfilled with some constant $N_{1}$ because of condition (b) on $\Theta$. Conditions (a)-(b) on $\widehat{\Theta}_{m}$ will be inherited from $\Theta_{m}$.

Secondly, Lemma 2.3 enables us to correct $\left\{\widehat{\Theta}_{m}\right\}$ so that condition (e) is obeyed as well.

The equivalence of $\rho(\cdot, \cdot)$ and $\hat{\rho}(\cdot, \cdot)$ is an easy consequence of the above construction.

Proposition 2.10. Let $\Theta$ be a continuous or semi-continuous or discrete ellipsoid cover of $\mathbb{R}^{n}$ and denote $B_{\rho}(x, r):=\left\{y \in \mathbb{R}^{n}: \rho(x, y)<r\right\}$. Then

$$
\left|B_{\rho}(x, r)\right| \sim r, \quad x \in \mathbb{R}^{n}, \quad r>0
$$

where the constants of equivalence depend only on $\mathbf{p}(\Theta)$. Therefore, the Lebesgue measure on $\mathbb{R}^{n}$ is a doubling measure with respect to the quasi-distance $\rho(\cdot, \cdot)$, i.e.

$$
\left|B_{\rho}(x, 2 r)\right| \leq c\left|B_{\rho}(x, r)\right|, \quad x \in \mathbb{R}^{n}, \quad r>0 .
$$

In other words, $\mathbb{R}^{n}$ equipped with the distance $\rho(\cdot, \cdot)$ defined by Definition 2.14 and the Lebesgue measure, is a space of homogeneous type.

Proof. We only consider the case when $\rho(\cdot, \cdot)$ is generated by a discrete ellipsoid cover of $\mathbb{R}^{n}$. We first prove that $\left|B_{\rho}(x, r)\right| \geq c r$. Choose $m$ so that $a_{2} 2^{-a_{o} m}<r \leq$ $a_{2} 2^{-a_{o}(m-1)}$. There exists $\theta \in \Theta_{m}$ such that $x \in \theta$ and hence by property (a) of $\bar{\Theta}$

$$
a_{1} 2^{-a_{o} m} \leq|\theta| \leq a_{2} 2^{-a_{o} m}<r \text {. }
$$

From this and the definition of $\rho(\cdot, \cdot)$ it follows that $\theta \subset B_{\rho}(x, r)$ and hence

$$
\left|B_{\rho}(x, r)\right| \geq|\theta| \geq a_{1} 2^{-a_{o} m} \geq\left(a_{1} / a_{2}\right) 2^{-a_{0}} r=c r .
$$

In the other direction, note first that

$$
B_{\rho}(x, r)=\bigcup_{\theta \in \Theta: x \in \theta,|\theta|<r} \theta .
$$

Suppose $\theta \in \Theta_{m}$ is of minimum level such that $x \in \theta$ and $|\theta|<r$. Let $\theta^{\prime} \in \Theta$ be any ellipsoid such that $x \in \theta^{\prime}$ and $\left|\theta^{\prime}\right|<r$. Then $\theta^{\prime} \in \Theta_{m+l}$ for some $l \geq 0$. Setting $\omega^{\prime}:=A_{\theta}^{-1}\left(\theta^{\prime}\right)$, one has, by property (b) of $\Theta$,

$$
\operatorname{diam} \omega^{\prime}=2\left\|M_{\theta}^{-1} M_{\theta^{\prime}}\right\|_{\ell_{2} \rightarrow \ell_{2}} \leq 2 a_{5} .
$$

Therefore, $A_{\theta}^{-1}\left(B_{\rho}(x, r)\right) \subset B\left(0,2 a_{5}\right)$ and hence $\left|A_{\theta}^{-1}\left(B_{\rho}(x, r)\right)\right| \leq c, c=c\left(n, a_{5}\right)$. This leads to

$$
\left|B_{\rho}(x, r)\right| \leq c\left|\operatorname{det} M_{\theta}\right|=c|\theta|<c r .
$$

\section{Construction of a multilevel system of Bases}

Given a discrete ellipsoid cover $\Theta$, we shall first construct for each level $m \in \mathbb{Z}$ a stable basis $\Phi_{m}$ whose elements are smooth piecewise polynomials supported on the ellipsoids of $\Theta_{m}$. Second, we shall utilize the bases $\left\{\Phi_{m}\right\}$ for the introduction of maps which locally preserve polynomials. 
3.1. Coloring the ellipsoids in $\Theta$. We begin by splitting the ellipsoid cover $\Theta$ into no more than $2 N_{1}$ disjoint subsets (colors) $\left\{\Theta^{\nu}\right\}_{\nu=1}^{2 N_{1}}$ so that neither two ellipsoids $\theta^{\prime}, \theta^{\prime \prime} \in \Theta_{m} \cup \Theta_{m+1}$ with $\theta^{\prime} \cap \theta^{\prime \prime} \neq \emptyset$ are of the same color. Indeed, using property (c) of $\Theta$ it is easy to color any level $\Theta_{m}$ by using no more than $N_{1}$ colors. So, we use at most $N_{1}$ colors to color the ellipsoids in $\left\{\Theta_{2 j}\right\}_{j \in \mathbb{Z}}$ and further at most $N_{1}$ colors to color the ellipsoids in $\left\{\Theta_{2 j+1}\right\}_{j \in \mathbb{Z}}$.

Thus we may assume that we have the following disjoint splitting

$$
\Theta=\bigcup_{\nu=1}^{2 N_{1}} \Theta^{\nu} \quad \text { and } \quad \Theta_{2 j}=\bigcup_{\nu=1}^{N_{1}} \Theta_{2 j}^{\nu}, \quad \Theta_{2 j+1}=\bigcup_{\nu=N_{1}+1}^{2 N_{1}} \Theta_{2 j+1}^{\nu}, \quad j \in \mathbb{Z},
$$

where if $\theta^{\prime} \in \Theta_{m_{1}}^{\nu_{1}}, \theta^{\prime \prime} \in \Theta_{m_{2}}^{\nu_{2}}$ with $\left|m_{1}-m_{2}\right| \leq 1$, and $\theta^{\prime} \cap \theta^{\prime \prime} \neq \emptyset$, then $\nu_{1} \neq \nu_{2}$.

3.2. Definition of single level bases. We first introduce $2 N_{1}$ smooth piecewise polynomial bumps associated with the colors from above. For fixed positive integers $L$ and $k(L \geq k)$ we define

$$
\phi_{\nu}(x):=\left(1-|x|^{2}\right)_{+}^{L+\nu k}, \quad \nu=1,2, \ldots, 2 N_{1}, \quad x_{+}:=\max \{x, 0\} .
$$

Notice that $\phi_{\nu} \in C^{L+\nu k-1} \subset C^{L}$.

For any $\theta \in \Theta$ we let $A_{\theta}$ denote the affine transform from Definition 2.1 such that $A_{\theta}\left(B^{*}\right)=\theta\left(\right.$ recall $\left.B^{*}:=B(0,1)\right)$ and set

$$
\phi_{\theta}:=\phi_{\nu} \circ A_{\theta}^{-1} \quad \text { if } \quad \theta \in \Theta^{\nu}, 1 \leq \nu \leq 2 N_{1} .
$$

In a standard way we introduce $m$ th level partitions of unity by defining for any $\theta \in \Theta_{m}$

$$
\varphi_{\theta}:=\frac{\phi_{\theta}}{\sum_{\theta^{\prime} \in \Theta_{m}} \phi_{\theta^{\prime}}} .
$$

By the properties of the ellipsoids from $\Theta$ it follows that

$$
\sum_{\theta \in \Theta_{m}} \varphi_{\theta}(x)=1 \quad \text { and } \quad 0<c_{1} \leq \sum_{\theta \in \Theta_{m}} \phi_{\theta}(x) \leq c_{2} \quad \text { for } x \in \mathbb{R}^{n} .
$$

Let

$$
\left\{P_{\beta}:|\beta|=\beta_{1}+\cdots+\beta_{n} \leq k-1\right\}, \quad \text { where } \operatorname{deg} P_{\beta}=|\beta|,
$$

be an orthonormal basis in $L_{2}\left(B^{*}\right)$ for the space $\mathcal{P}_{k}$ of all polynomials in $n$ variables of total degree $k-1$. Since $\left\|P_{\beta}\right\|_{L_{2}\left(B^{*}\right)}=1$, then $\left\|P_{\beta}\right\|_{L_{\infty}\left(B^{*}\right)} \sim 1$ and hence

$$
\left\|P_{\beta} \phi_{\nu}\right\|_{L_{2}\left(B^{*}\right)} \sim\left\|P_{\beta} \phi_{\nu}\right\|_{L_{\infty}\left(B^{*}\right)} \sim 1 \text {. }
$$

For any $\theta \in \Theta$ and $|\beta|<k$ we define

$$
P_{\theta, \beta}:=|\theta|^{-1 / 2} P_{\beta} \circ A_{\theta}^{-1}
$$

and

$$
g_{\theta, \beta}:=\varphi_{\theta} P_{\theta, \beta} .
$$

Notice that $\left\|g_{\theta, \beta}\right\|_{2} \sim 1$ and in general $\left\|g_{\theta, \beta}\right\|_{p} \sim|\theta|^{1 / p-1 / 2}(0<p \leq \infty)$; also $g_{\theta, \beta} \in C^{L}$. Now we define the $m$ th level basis $\Phi_{m}$ by

$$
\Phi_{m}:=\left\{g_{\theta, \beta}: \theta \in \Theta_{m},|\beta| \leq k-1\right\}
$$

and set

$$
S_{m}:=\operatorname{span}\left(\Phi_{m}\right)
$$


i.e. $S_{m}$ is the set of all functions $f$ on $\mathbb{R}^{n}$ of the form

$$
f(x)=\sum_{\theta \in \Theta_{m},|\beta|<k} c_{\theta, \beta} g_{\theta, \beta}(x), \quad x \in \mathbb{R}^{n},
$$

where $\left\{c_{\theta, \beta}\right\}$ is an arbitrary collection of real numbers. Notice that the sum in (3.12) is finite for any $x \in \mathbb{R}^{n}$ and hence well defined. Also, each $f \in S_{m}$ is a piecewise polynomial over the set of all intersections $\theta \cap \theta^{\prime}, \theta, \theta^{\prime} \in \Theta_{m}$.

Remark 3.1. (a) By the definition of $g_{\theta, \beta}$ it readily follows that $\mathcal{P}_{k} \subset S_{m}$.

(b) $\Phi_{m}$ is linearly independent, i.e. if $\sum_{\theta \in \Theta_{m},|\beta|<k} c_{\theta, \beta} g_{\theta, \beta}=0$ a.e., then $c_{\theta, \beta}=0 \forall \theta \in \Theta_{m}, \forall|\beta|<k$. More importantly, $\Phi_{m}$ is in a sense locally linearly independent and $L_{p}$ stable, which will be established in the following theorem.

Theorem 3.2. Any function $f \in S_{m}$ has a unique representation

$$
f(x)=\sum_{\theta \in \Theta_{m},|\beta|<k}\left\langle f, \tilde{g}_{\beta, \theta}\right\rangle g_{\theta, \beta}(x),
$$

where for every $x \in \mathbb{R}^{n}$ the sum is finite and the functions $\tilde{g}_{\beta, \theta}$ have he following properties: For every $\theta \in \Theta_{m}$ there exists an ellipsoid $B_{\theta}:=A_{\theta}\left(B_{\theta}^{*}\right) \subset \theta$ for some ball $B_{\theta}^{*} \subset B^{*}$ with $\left|B_{\theta}\right| \sim|\theta|$ such that for $0<p \leq \infty$

$$
\left\langle g_{\theta^{\prime}, \beta^{\prime}}, \tilde{g}_{\theta, \beta}\right\rangle=\delta_{\theta, \theta^{\prime}} \delta_{\theta, \theta^{\prime}}, \quad \operatorname{supp} \tilde{g}_{\theta, \beta} \subset \bar{B}_{\theta}, \quad\left\|\tilde{g}_{\theta, \beta}\right\|_{p} \sim|\theta|^{1 / p-1 / 2} .
$$

Moreover, if $f \in S_{m} \cap L_{p}, 0<p \leq \infty$, and $f=\sum_{\theta \in \Theta_{m},|\beta|<k} c_{\theta, \beta} g_{\theta, \beta}$, then

$$
\|f\|_{p} \sim\left(\sum_{\theta \in \Theta_{m},|\beta|<k}\left\|c_{\theta, \beta} g_{\theta, \beta}\right\|_{p}^{p}\right)^{1 / p}
$$

with the obvious modification when $p=\infty$. Here all constants of equivalence depend only on $\mathbf{p}(\Theta), L, p$, and $k$.

Proof. We first construct the balls $B_{\theta}^{*} \subset B^{*}$. Fix $\theta \in \Theta_{m}(m \in \mathbb{Z})$ and let $\mathcal{X}_{\theta}$ be the set of all $\theta^{\prime} \in \Theta_{m}$ such that $\theta^{\prime} \cap \theta \neq \emptyset$. Denote

$$
\mathcal{X}_{\theta}^{*}:=\left\{A_{\theta}^{-1}\left(\theta^{\prime}\right): \theta^{\prime} \in \mathcal{X}_{\theta}\right\} .
$$

By the properties of $\Theta$, for any $\eta \in \mathcal{X}_{\theta}^{*}$ we have $\left|\eta \cap B^{*}\right| \geq a_{8}\left|B^{*}\right|=c>0$ and there exist balls $B_{1}, B_{2}$ such that $B_{1} \subset \eta \subset B_{2}$ and $\left|B_{1}\right| \sim\left|B_{2}\right| \sim 1$. From this and because $\# \mathcal{X}_{\theta}^{*} \leq N_{1}$ it follows that there exists a ball $B_{\theta}^{*} \subset B^{*}$ such that $\left|B_{\theta}^{*}\right| \sim 1$ and for each $\eta \in \mathcal{X}_{\theta}^{*}$ either $B_{\theta}^{*} \subset \eta$ or $B_{\theta}^{*} \cap \eta=\emptyset$. Define $B_{\theta}:=A_{\theta}\left(B_{\theta}^{*}\right)$.

Denote by $\mathcal{Y}_{\theta}$ the set of all $\theta^{\prime} \in \mathcal{X}_{\theta}$ such that $B_{\theta} \subset \theta^{\prime}$ and set

$$
\mathcal{F}_{\theta}:=\left\{g_{\theta^{\prime}, \beta}^{\diamond}:=g_{\theta^{\prime}, \beta} \mathbb{1}_{B_{\theta}}: \theta^{\prime} \in \mathcal{Y}_{\theta},|\beta|<k\right\} .
$$

It is an important observation that the set of functions $\mathcal{F}_{\theta}$ is linearly independent. Indeed, since all ellipsoids $\theta^{\prime} \in \mathcal{Y}_{\theta}$ contain $B_{\theta}$, every two of them have distinct colors. Thus if $\theta^{\prime} \in \mathcal{Y}_{\theta}$ and $\theta^{\prime} \in \Theta_{m}^{\nu_{j}}$ for some $1 \leq \nu_{j} \leq 2 N_{1}$, then $\phi_{\theta^{\prime}} P_{\theta^{\prime}, \beta^{\prime}}$ is a polynomial of degree exactly $L+\nu_{j} k+\left|\beta^{\prime}\right|$ on $B_{\theta}$ and $L+\nu_{j} k \leq L+\nu_{j} k+\left|\beta^{\prime}\right|<$ $L+\nu_{j}(k+1)$. Consequently, the functions $\left\{\phi_{\theta^{\prime}} P_{\theta^{\prime}, \beta} \mathbb{1}_{B_{\theta}}: \theta^{\prime} \in \mathcal{Y}_{\theta},|\beta|<k\right\}$ are linearly independent on $B_{\theta}$ and hence $\mathcal{F}_{\theta}$ is linearly independent.

Define $g_{\theta^{\prime}, \beta}^{*}:=|\theta|^{1 / 2} g_{\theta^{\prime}, \beta}^{\diamond} \circ A_{\theta}$. Notice that $\operatorname{supp} g_{\theta^{\prime}, \beta}^{*}=\overline{B_{\theta}^{*}}$ and $\left\|g_{\theta^{\prime}, \beta}^{*}\right\|_{2} \sim$ $\left\|g_{\theta^{\prime}, \beta}^{*}\right\|_{\infty} \sim 1$. Let

$$
\mathcal{F}_{\theta}^{*}:=\left\{g_{\theta^{\prime}, \beta}^{*}: \theta^{\prime} \in \mathcal{Y}_{\theta},|\beta|<k\right\} \quad \text { and } \quad \Lambda_{\theta}:=\left\{\lambda:=\left(\theta^{\prime}, \beta\right): \theta^{\prime} \in \mathcal{Y}_{\theta},|\beta|<k\right\} .
$$


As $\mathcal{F}_{\theta}$ is linearly independent, $\mathcal{F}_{\theta}^{*}$ is linearly independent as well. Consequently, the Gram matrix

$$
G_{\theta}:=\left(\left\langle g_{\theta^{\prime}, \beta^{\prime}}^{*}, g_{\theta^{\prime \prime}, \beta^{\prime \prime}}^{*}\right\rangle\right)_{\left(\theta^{\prime}, \beta^{\prime}\right),\left(\theta^{\prime \prime}, \beta^{\prime \prime}\right) \in \Lambda_{\theta}}
$$

is nonsingular and hence its inverse

$$
G_{\theta}^{-1}=:\left(R_{\left(\theta^{\prime}, \beta^{\prime}\right),\left(\theta^{\prime \prime}, \beta^{\prime \prime}\right)}\right)_{\left(\theta^{\prime}, \beta^{\prime}\right),\left(\theta^{\prime \prime}, \beta^{\prime \prime}\right) \in \Lambda_{\theta}}
$$

exists.

We next show that the functions

$$
\tilde{g}_{\theta, \beta}:=\sum_{\left(\theta^{\prime}, \beta^{\prime}\right) \in \Lambda_{\theta}} R_{(\theta, \beta),\left(\theta^{\prime}, \beta^{\prime}\right)} g_{\theta^{\prime}, \beta^{\prime}}^{\diamond}
$$

form a dual system to $\Phi_{m}$. Indeed, if $\theta^{\prime} \in \Theta_{m}$ and $\theta^{\prime} \notin \mathcal{Y}_{\theta}$, then $\theta^{\prime} \cap \theta=\emptyset$ and hence $\left\langle g_{\theta^{\prime}, \beta^{\prime}}, \tilde{g}_{\theta, \beta}\right\rangle=0$. If $\theta^{\prime} \in \mathcal{Y}_{\theta}$ and $\left|\beta^{\prime}\right|<k$, then

$$
\begin{aligned}
\left\langle g_{\theta^{\prime}, \beta^{\prime}}, \tilde{g}_{\theta, \beta}\right\rangle & =|\theta|\left\langle g_{\theta^{\prime}, \beta^{\prime}} \circ A_{\theta}, \tilde{g}_{\theta, \beta} \circ A_{\theta}\right\rangle=\sum_{\left(\theta^{\prime \prime}, \beta^{\prime \prime}\right) \in \Lambda_{\theta}} R_{(\theta, \beta),\left(\theta^{\prime \prime}, \beta^{\prime \prime}\right)}\left\langle g_{\theta^{\prime}, \beta^{\prime}}^{*}, g_{\theta^{\prime \prime}, \beta^{\prime \prime}}^{*}\right\rangle \\
& =\left(G_{\theta}^{-1} G_{\theta}\right)_{(\theta, \beta),\left(\theta^{\prime}, \beta^{\prime}\right)}=\delta_{(\theta, \beta),\left(\theta^{\prime}, \beta^{\prime}\right)}
\end{aligned}
$$

as claimed.

Our next and most important step is to show that

$$
\left|R_{\left(\theta^{\prime}, \beta^{\prime}\right),\left(\theta^{\prime \prime}, \beta^{\prime \prime}\right)}\right| \leq c \quad \forall\left(\theta^{\prime}, \beta^{\prime}\right),\left(\theta^{\prime \prime}, \beta^{\prime \prime}\right) \in \Lambda_{\theta},
$$

where $c>0$ depends only on $\mathbf{p}(\Theta), L$, and $k$. We shall use a compactness argument.

It is readily seen that the set $\mathcal{F}_{\theta}^{*}$ is a particular case of the general case when we have a collection of linearly independent functions

$$
\mathcal{F}=\left\{f_{j, \beta}:=\frac{\left(\phi_{\nu_{j}} P_{\beta}\right) \circ L_{j}}{\sum_{j=1}^{J} \phi_{\nu_{j}} \circ L_{j}} \cdot \mathbb{1}_{B_{0}}: j=1,2, \ldots, J,|\beta|<k\right\},
$$

where $B_{0} \subset B^{*}$ is a ball with $\left|B_{0}\right| \geq c_{1}>0$, the indices

$$
1 \leq \nu_{1}<\nu_{2}<\cdots<\nu_{J} \leq N_{1}
$$

are fixed, $\phi_{\nu}$ are from (3.2) and $P_{\beta}$ are as in (3.6) with the normalization from (3.7), i.e. $\left\|P_{\beta}\right\|_{2}=1$, which implies $\left\|\phi_{\nu} P_{\beta}\right\|_{\infty} \sim 1$. We also assume that $L_{j}$, $j=1,2, \ldots, J$, are affine transforms of the form $L_{j}(x)=M_{j} x+v_{j}$ satisfying the following conditions:

(i) $M_{j}=U_{j} D_{j} V_{j}$, where $U_{j}$ and $V_{j}$ are orthogonal $n \times n$ matrices, $D_{j}=\operatorname{diag}\left(\sigma_{1}^{j}, \sigma_{2}^{j}, \ldots, \sigma_{n}^{j}\right)$ with $0<c_{2} \leq \min _{\ell} \sigma_{\ell}^{j} \leq \max _{\ell} \sigma_{\ell}^{j} \leq c_{3}$, and $\left|v_{j}\right| \leq c_{4}$;

(ii) $0<c_{5} \leq \sum_{j=1}^{J}\left(\phi_{\nu_{j}} \circ L_{j}\right)(x) \leq c_{6} \quad$ for $x \in B^{*}$;

(iii) $L_{j}\left(B_{0}\right) \subset B^{*}$.

Let $\Lambda:=\{\lambda:=(j, \beta): j=1,2, \ldots, J,|\beta|<k\}$. Since $F$ is linearly independent, the Gram matrix $G:=\left(\left\langle f_{\lambda}, f_{\lambda^{\prime}}\right\rangle\right)_{\lambda, \lambda^{\prime} \in \Lambda}$ is nonsingular and hence $G^{-1}=:\left(R_{\lambda, \lambda^{\prime}}\right)_{\lambda, \lambda^{\prime} \in \Lambda}$ exists.

Each of the affine transforms $L_{j}$ depends on parameters from a subset, say, $K$ of the set $\mathbb{R}^{n \times n} \times \mathbb{R}^{n} \times \mathbb{R}^{n \times n} \times \mathbb{R}^{n}$. The set of all orthogonal $n \times n$ matrices is a compact subset of $\mathbb{R}^{n \times n}$. Hence the parameters of all affine transforms $L_{j}$ satisfying 
condition (i) belong to a compact subset, say, $K_{1}$ of $\mathbb{R}^{n \times n} \times \mathbb{R}^{n} \times \mathbb{R}^{n \times n} \times \mathbb{R}^{n}$. On the other hand, condition (iii) on $L_{j}$ can be expressed in the form

$$
\max _{\left|x-x_{0}\right| \leq a}\left|M_{j} x+v_{j}\right| \leq 1
$$

where $x_{0}$ and $a(a \sim 1)$ are the center and radius of $B_{0}$. Therefore, conditions (ii) and (iii) define $K$ as a closed subset of the compact $K_{1}$ and hence $K$ is compact.

The entries of $G$ and $\operatorname{det} G$ apparently depend continuously on the parameters of the affine transforms $L_{j}, j=1,2, \ldots, J$, and since $K$ is compact

$$
\left|\left\langle f_{\lambda}, f_{\lambda^{\prime}}\right\rangle\right| \leq c_{7} \quad \forall \lambda, \lambda^{\prime} \in \Lambda \quad \text { and } \quad 0<c_{8} \leq \operatorname{det} G \leq c_{9} .
$$

From this it follows that

$$
\left|R_{\lambda, \lambda^{\prime}}\right| \leq c_{10} \quad \forall \lambda, \lambda^{\prime} \in \Lambda,
$$

where $c_{10}$ as well as $c_{7}, c_{8}$, and $c_{9}$ depends only on $c_{1}, \ldots, c_{6}, \mathbf{p}(\Theta), L$, and $k$. Finally, Using that there are only finitely many possibilities for the indices $1 \leq$ $\nu_{1}<\nu_{2}<\cdots<\nu_{J} \leq N_{1}$ we conclude that the constant $c_{10}$ in estimate (3.18) can be selected independently of these indices.

Applying the above claim to the specific case at hand it follows that estimate (3.17) holds. Then (3.14) follows by (3.16) and (3.17).

The stability properties (3.15) follow by a standard argument from properties (3.14) of the dual.

Let us now introduce the more compact notation

$$
\Lambda_{m}:=\left\{\lambda:=(\theta, \beta): \theta \in \Theta_{m},|\beta|<k\right\},
$$

and if $\lambda:=(\theta, \beta)$ we shall denote by $\theta_{\lambda}$ and $\beta_{\lambda}$ the components of $\lambda$.

We denote by $\widetilde{\Phi}_{m}:=\left\{\tilde{g}_{\lambda}: \lambda \in \Lambda_{m}\right\}$ the dual system introduced in Theorem 3.2, i.e. $\left\langle g_{\lambda^{\prime}}, \tilde{g}_{\lambda}\right\rangle=\delta_{\lambda, \lambda^{\prime}}$ for $\lambda, \lambda^{\prime} \in \Lambda_{m}$.

3.3. Local projectors onto polynomials. The anisotropic regularity notions we are aiming at will rely on appropriate multilevel decompositions of functions based on a given ellipsoid cover. These decompositions, in turn, will be obtained with the aid of appropriate operators which map $L_{p}^{\text {loc }}$ into $S_{m}$ and locally preserve $\mathcal{P}_{k}$ and hence provide good local approximation.

Recall first the definition of local and global moduli of smoothness that are standard means to describe the quality of approximation. The usual forward differences of $f$ in direction $h \in \mathbb{R}^{n}$ on a set $E \subset \mathbb{R}^{n}$ are defined by $\Delta_{h} f(x):=\Delta_{h}^{1} f(x):=$ $f(x+h)-f(x)$ if the line segment $[x, x+h]$ is contained in $E$ and by $\Delta_{h} f(x):=0$ otherwise. Inductively, we set

$$
\Delta_{h}^{k} f(x):=\Delta_{h}\left(\Delta_{h}^{k-1} f(x)\right) \quad \text { if }[x, x+k h] \subset E \quad \text { and } \quad \Delta_{h}^{k} f(x):=0 \text { otherwise. }
$$

Then the $k$ th $L_{p}$-moduli of smoothness on $\theta$ and $\mathbb{R}^{n}$ are defined by

$$
\omega_{k}(f, \theta)_{p}:=\sup _{h \in \mathbb{R}^{n}}\left\|\Delta_{h}^{k} f\right\|_{L_{p}(\theta)} \quad \text { and } \quad \omega_{k}(f, t)_{p}:=\sup _{|h| \leq t}\left\|\Delta_{h}^{k} f\right\|_{p}, \quad t>0 .
$$

The form of the operators will differ somewhat for $p \geq 1$ and $p<1$.

(a) The case $1 \leq \boldsymbol{p} \leq \infty$. There are actually a number of ways to construct suitable operators. While the basic principles are known some of the estimates to be used are less familiar. Since these notions are essential for what follows we sketch the arguments for the reader's convenience. A first natural idea is to use the bases 
$\left\{\Phi_{m}\right\}$ and their duals $\left\{\widetilde{\Phi}_{m}\right\}$ to introduce projectors mapping $L_{p}^{\text {loc }}$ onto the spaces $S_{m}$ by

$$
Q_{m} f:=\sum_{\lambda \in \Lambda_{m}}\left\langle f, \tilde{g}_{\lambda}\right\rangle g_{\lambda}
$$

with $\tilde{g}_{\lambda}$ for $\lambda=(\theta, \beta)$ from (3.14).

Alternatively, simpler local projectors onto polynomials are obtained as follows. As in (3.6) suppose $\left\{P_{\beta}:|\beta| \leq k-1\right\}$ is an arbitrary (but fixed) orthonormal basis for $\mathcal{P}_{k}$ in $L_{2}\left(B^{*}\right)$. Then for any $\theta \in \Theta$

$$
\left\{P_{\theta, \beta}:=|\theta|^{-1 / 2} P_{\beta} \circ A_{\theta}^{-1}:|\beta|<k\right\}
$$

is an orthonormal basis for $\mathcal{P}_{k}$ in $L_{2}(\theta)$. Using again our compact notation from (3.9), we can write for $g_{\theta, \beta}:=\varphi_{\theta} P_{\theta, \beta}, P_{\lambda}:=P_{\theta, \beta}$, and $g_{\lambda}:=g_{\theta, \beta}, \lambda:=(\theta, \beta)$. We define

$$
T_{m} f:=\sum_{\theta \in \Theta_{m}} \sum_{|\beta| \leq k-1}\left\langle f, P_{\theta, \beta}\right\rangle g_{\theta, \beta}=\sum_{\lambda \in \Lambda_{m}}\left\langle f, P_{\lambda}\right\rangle g_{\lambda}
$$

where $\Lambda_{m}$ is given by (3.19).

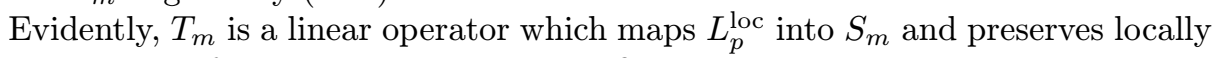
all polynomials from $\mathcal{P}_{k}$. To be more specific, setting

$$
\theta^{*}:=\cup\left\{\theta^{\prime} \in \Theta_{m}: \theta \cap \theta^{\prime} \neq \emptyset\right\} \quad \text { for } \theta \in \Theta_{m},
$$

it is easy to see that if $\left.f\right|_{\theta^{*}}=\left.P\right|_{\theta^{*}}$ with $P \in \mathcal{P}_{k}$, then $\left.T_{m} f\right|_{\theta}=\left.P\right|_{\theta}$.

(b) The case $0<\boldsymbol{p}<\mathbf{1}$. Apparently the above operators are no longer usable, when working in $L_{p}$ with $p<1$. Hence, we need to modify them. In fact, the following construction would cover the full range $0<p \leq \infty$. For $0<p \leq \infty$ and a given ellipsoid $\theta \in \Theta$, we let $T_{\theta, p}:\left.L_{p}(\theta) \rightarrow \mathcal{P}_{k}\right|_{\theta}$ be a projector such that

$$
\left\|f-T_{\theta, p} f\right\|_{L_{p}(\theta)} \leq c \omega_{k}(f, \theta)_{p}, \quad f \in L_{p}(\theta),
$$

where $c>0$ depends only on $\mathbf{p}(\Theta), L, k$, and $p$. Note that $T_{\theta, p} f$ can simply be defined as the best (or near best) approximation to $f$ from $\mathcal{P}_{k}$ in $L_{p}(\theta)$. Then (3.25) is a consequence of Whitney's theorem. Also, $T_{\theta, p}$ can be realized as a linear projector onto $\left.\mathcal{P}_{k}\right|_{\theta}$ if $p \geq 1$ by using, say, the Averaged Taylor polynomials, see e.g. [10]. Of course, $T_{\theta, p}$ will be a nonlinear operator if $p<1$.

We now define the operator $T_{m, p}: L_{p}^{\text {loc }} \rightarrow S_{m}$ by

$$
T_{m, p} f:=\sum_{\theta \in \Theta_{m}} \varphi_{\theta}(x) T_{\theta, p} f .
$$

Evidently, the operator $T_{m, p}(0<p \leq \infty)$ is a local projector onto $\mathcal{P}_{k}$ (nonlinear if $p<1$ ) just like $T_{m}$. Since $T_{m, p} f \in S_{m}$, it can be represented in terms of the basis functions $g_{\lambda}$ as

$$
T_{m, p} f:=\sum_{\theta \in \Theta_{m}} \sum_{|\beta|<k} b_{\theta, \beta}(f) g_{\theta, \beta}=\sum_{\lambda \in \Lambda_{m}} b_{\lambda}(f) g_{\lambda},
$$

where $b_{\lambda}(f):=\left\langle T_{m, p} f, \tilde{g}_{\lambda}\right\rangle$ depends nonlinearly on $f$ if $p<1$. 
In summary, any $\hat{T}_{m} \in\left\{Q_{m}, T_{m}, T_{m, p}\right\}$ defined by (3.21), (3.23), or (3.27), has the representation

$$
\hat{T}_{m} f=\sum_{\lambda \in \Lambda_{m}} b_{\lambda}(f) g_{\lambda}, \text { where } b_{\lambda}(f)=\left\{\begin{array}{lll}
\left\langle f, \tilde{g}_{\lambda}\right\rangle & \text { if } & \hat{T}_{m}=Q_{m} \\
\left\langle f, P_{\lambda}\right\rangle & \text { if } & \hat{T}_{m}=T_{m}, \\
\left\langle T_{m, p} f, \tilde{g}_{\lambda}\right\rangle & \text { if } & \hat{T}_{m}=T_{m, p}
\end{array}\right.
$$

We next record the most important properties of the operators $Q_{m}, T_{m}$, and $T_{m, p}$ from above.

Lemma 3.3. Assume that $0<p \leq \infty$ and let $\hat{T}_{m}$ be the operator $Q_{m}$ from (3.21) or $T_{m}$ from (3.23) or $T_{m, p}$ from (3.26) if $1 \leq p \leq \infty$, and $\hat{T}_{m}:=T_{m, p}$ if $0<p<1$. Then for $f \in L_{p}^{\text {loc }}$ and $\theta \in \Theta_{m}(m \in \mathbb{Z})$

$$
\left\|\hat{T}_{m} f\right\|_{L_{p}(\theta)} \leq c\|f\|_{L_{p}\left(\theta^{*}\right)}
$$

and

$$
\left\|f-\hat{T}_{m} f\right\|_{L_{p}(\theta)} \leq c \sum_{\theta^{\prime} \in \Theta_{m}: \theta^{\prime} \cap \theta \neq \emptyset} \omega_{k}\left(f, \theta^{\prime}\right)_{p} .
$$

Furthermore, if $f \in L_{p}^{\text {loc }}$

$$
\left\|f-\hat{T}_{m} f\right\|_{L_{p}(K)} \rightarrow 0 \quad \text { as } m \rightarrow \infty \quad \text { for any compact } K \subset \mathbb{R}^{n},
$$

and if $f \in L_{p}\left(L_{\infty}:=C_{0}\right)$

$$
\left\|f-\hat{T}_{m} f\right\|_{p} \rightarrow 0 \quad \text { as } m \rightarrow \infty .
$$

Proof. We first prove (3.29) in the case $\hat{T}_{m}=Q_{m}$ and $1 \leq p \leq \infty$ (the proof in the other cases is similar). By (3.21) and (3.14) it follows that

$$
\begin{aligned}
\left\|Q_{m} f\right\|_{L_{p}(\theta)} & \leq \sum_{\lambda \in \Lambda_{m}: \theta_{\lambda} \cap \theta \neq \emptyset}\left|\left\langle f, \tilde{g}_{\lambda}\right\rangle\right|\left\|g_{\lambda}\right\|_{p} \\
& \leq \sum_{\lambda \in \Lambda_{m}: \theta_{\lambda} \cap \theta \neq \emptyset}\|f\|_{L_{p}\left(\theta_{\lambda}\right)}\left\|\tilde{g}_{\lambda}\right\|_{p^{\prime}}\left\|g_{\lambda}\right\|_{p} \leq c \sum_{\lambda \in \Lambda_{m}: \theta_{\lambda} \cap \theta \neq \emptyset}\|f\|_{L_{p}\left(\theta_{\lambda}\right)} \\
& \leq c\|f\|_{L_{p}\left(\theta^{*}\right)}\left(1 / p+1 / p^{\prime}=1\right)
\end{aligned}
$$

as desired.

The proof of (3.30) relies on a Whitney type estimate that will be given first. For a given set $E \subset \mathbb{R}^{n}$, denote by $E_{k}(f, E)_{p}$ the best $L_{p}(E)$ approximation of $f$ from $\mathcal{P}_{k}$, i.e.

$$
E_{k}(f, E)_{p}:=\inf _{P \in \mathcal{P}_{k}}\|f-P\|_{L_{p}(E)} .
$$

We claim now that for $0<p \leq \infty$ and any $\theta \in \Theta_{m}$

$$
E_{k}\left(f, \theta^{*}\right)_{p} \leq c_{p} \sum_{\theta^{\prime} \in \mathcal{X}_{\theta}} \omega_{k}\left(f, \theta^{\prime}\right)_{p}
$$

where $\theta^{*}$ is defined in (3.24) and $\mathcal{X}_{\theta}:=\left\{\theta^{\prime} \in \Theta_{m}: \theta^{\prime} \cap \theta \neq \emptyset\right\}$. Indeed, by Whitney's estimate for a ball it follows by an affine transform that $E_{k}(f, \theta)_{p} \leq c \omega_{k}(f, \theta)_{p}$ for any ellipsoid $\theta$. For any $\theta^{\prime} \in \mathcal{X}_{\theta}$, let $P_{\theta^{\prime}} \in \mathcal{P}_{k}$ be such that $\left\|f-P_{\theta^{\prime}}\right\|_{L_{p}\left(\theta^{\prime}\right)}=$ $E_{k}\left(f, \theta^{\prime}\right)_{p}$. Then using condition (e) on discrete covers we get

$$
\begin{aligned}
\left\|P_{\theta^{\prime}}-P_{\theta}\right\|_{L_{p}\left(\theta^{\prime}\right)} & \leq c\left\|P_{\theta^{\prime}}-P_{\theta}\right\|_{L_{p}\left(\theta^{\prime} \cap \theta\right)} \leq c\left\|f-P_{\theta^{\prime}}\right\|_{L_{p}\left(\theta^{\prime} \cap \theta\right)}+c\left\|f-P_{\theta}\right\|_{L_{p}\left(\theta^{\prime} \cap \theta\right)} \\
& \leq c\left\|f-P_{\theta^{\prime}}\right\|_{L_{p}\left(\theta^{\prime}\right)}+c\left\|f-P_{\theta}\right\|_{L_{p}(\theta)} \leq c \omega_{k}\left(f, \theta^{\prime}\right)_{p}+c \omega_{k}(f, \theta)_{p} .
\end{aligned}
$$


By condition (c) on discrete covers, we know that $\# \mathcal{X}_{\theta} \leq N_{1}$. From this and the preceding estimate we conclude that

$$
\begin{aligned}
\left\|f-P_{\theta}\right\|_{L_{p}\left(\theta^{*}\right)} & \leq c \sum_{\theta^{\prime} \in \mathcal{X}_{\theta}}\left\|f-P_{\theta}\right\|_{L_{p}\left(\theta^{\prime}\right)} \leq c \sum_{\theta^{\prime} \in \mathcal{X}_{\theta}}\left\|P_{\theta^{\prime}}-P_{\theta}\right\|_{L_{p}\left(\theta^{\prime}\right)} \\
& +c \sum_{\theta^{\prime} \in \mathcal{X}_{\theta}}\left\|f-P_{\theta^{\prime}}\right\|_{L_{p}\left(\theta^{\prime}\right)} \leq c \sum_{\theta^{\prime} \in \mathcal{X}_{\theta}} \omega_{k}\left(f, \theta^{\prime}\right)_{p}
\end{aligned}
$$

which yields (3.34).

To prove (3.30) when $\hat{T}_{m}$ is $Q_{m}$ from (3.21) or $T_{m}$ from (3.23) and $1 \leq p \leq \infty$, we pick $P^{*} \in \mathcal{P}_{k}$ so that $\left\|f-P^{*}\right\|_{L_{p}\left(\theta^{*}\right)}$ realizes the estimate in (3.34), i.e.

$$
\left\|f-P^{*}\right\|_{L_{p}\left(\theta^{*}\right)} \leq c \sum_{\theta^{\prime} \in \mathcal{X}_{\theta}} \omega_{k}\left(f, \theta^{\prime}\right)_{p} .
$$

Then using that $\hat{T}_{m}\left(P^{*}\right)=P^{*}$ and $(3.29)$ we get

$$
\left\|f-\hat{T}_{m} f\right\|_{L_{p}(\theta)} \leq\left\|f-P^{*}\right\|_{L_{p}(\theta)}+\left\|\hat{T}_{m}\left(P^{*}-f\right)\right\|_{L_{p}(\theta)} \leq c\left\|f-P^{*}\right\|_{L_{p}\left(\theta^{*}\right)}
$$

and (3.30) follows.

We now establish (3.30) when $\hat{T}_{m}:=T_{m, p}$. We pick $P^{*} \in \mathcal{P}_{k}$ as above and using that $T_{m, p} P^{*}=P^{*}$ and (3.26) we get

$$
P^{*}-T_{m, p} f=T_{m, p} P^{*}-T_{m, p} f=\sum_{\theta \in \Theta_{m}} \varphi_{\theta}\left(P^{*}-T_{\theta, p} f\right) .
$$

Hence

$$
\begin{aligned}
\| f- & T_{m, p} f\left\|_{L_{p}(\theta)} \leq c\right\| f-P^{*}\left\|_{L_{p}(\theta)}+c\right\| P^{*}-T_{m, p} f \|_{L_{p}(\theta)} \\
& \leq c\left\|f-P^{*}\right\|_{L_{p}(\theta)}+c \sum_{\theta^{\prime} \in \Theta_{m}: \theta^{\prime} \cap \theta \neq \emptyset}\left\|P^{*}-T_{\theta^{\prime}, p} f\right\|_{L_{p}\left(\theta^{\prime}\right)} \\
& \leq c\left\|f-P^{*}\right\|_{L_{p}(\theta)}+c \sum_{\theta^{\prime} \in \Theta_{m}: \theta^{\prime} \cap \theta \neq \emptyset}\left(\left\|f-P^{*}\right\|_{L_{p}\left(\theta^{\prime}\right)}+\left\|f-T_{\theta^{\prime}, p} f\right\|_{L_{p}\left(\theta^{\prime}\right)}\right),
\end{aligned}
$$

which combined with (3.25) and (3.36) yields (3.30).

As was already mentioned in $\S 2.1$ (Remark 7 ), for any compact $K \subset \mathbb{R}^{n}$

$$
\max \left\{\operatorname{diam} \theta: \theta \in \Theta_{m}, \theta \cap K \neq \emptyset\right\} \rightarrow 0 \quad \text { as } m \rightarrow \infty .
$$

This and (3.30) readily imply (3.31), which leads to (3.32).

\section{TWO-LEVEL-SPLIT BASES}

In this section we assume that $T_{j}(j \in \mathbb{Z})$ is one of the operators $Q_{j}$ or $T_{j}$ or $T_{j, p}$, if $p \geq 1$, and $T_{j}:=T_{j, p}$, if $p<1$, defined in $\S 3.3$. We shall use these operators and the bases $\left\{\Phi_{j}\right\}_{j \in \mathbb{Z}}$ from (3.10) to define two-level-split bases which will play an important role in our further development. Further, we shall make use of the following representation of consecutive level polynomial bases, defined in (3.8):

$$
P_{\theta, \alpha}=: \sum_{|\beta|<k} m_{\alpha, \beta}^{\theta, \eta} P_{\eta, \beta}, \quad \theta \in \Theta_{j}, \quad \eta \in \Theta_{j+1} .
$$


Then since $\sum_{\eta \in \Theta_{j+1}} \varphi_{\eta}=1$, we have

$$
P_{\theta, \alpha}=\sum_{\eta \in \Theta_{j+1}: \theta \cap \eta \neq \emptyset|\beta|<k} \sum_{\alpha, \beta}^{\theta, \eta} P_{\eta, \beta} \varphi_{\eta} \quad \text { on } \theta .
$$

This yields

$$
\begin{aligned}
T_{j+1} f-T_{j} f= & \sum_{\eta \in \Theta_{j+1}} \sum_{|\beta|<k} b_{\eta, \beta}(f) P_{\eta, \beta} \varphi_{\eta}-\sum_{\theta \in \Theta_{j}} \sum_{|\alpha|<k} b_{\theta, \alpha}(f) P_{\theta, \alpha} \varphi_{\theta} \\
= & \sum_{\theta \in \Theta_{j}} \varphi_{\theta} \sum_{\eta \in \Theta_{j+1}} \sum_{|\beta|<k} b_{\eta, \beta}(f) P_{\eta, \beta} \varphi_{\eta} \\
& -\sum_{\theta \in \Theta_{j}} \sum_{|\alpha|<k} b_{\theta, \alpha}(f) \sum_{\theta \cap \eta \neq \emptyset|\beta|<k} \sum_{\beta, \alpha}^{\theta, \eta} P_{\eta, \beta} \varphi_{\theta} \varphi_{\eta} \\
= & \sum_{\eta \in \Theta_{j+1}} \sum_{\theta: \theta \cap \eta \neq \emptyset|\beta|<k} \sum_{\left.\mid b_{\eta, \beta}(f)-\sum_{|\alpha|<k} m_{\beta, \alpha}^{\theta, \eta} b_{\theta, \alpha}(f)\right\} P_{\eta, \beta} \varphi_{\eta} \varphi_{\theta},},
\end{aligned}
$$

where $b_{\lambda}(f)$ is given by (3.28), depending of the choice of $T_{m}$. Thus, setting

$$
\mathcal{M}_{j}:=\left\{\mu=(\eta, \theta, \beta): \eta \in \Theta_{j+1}, \theta \in \Theta_{j}, \theta \cap \eta \neq 0,|\beta|<k\right\}, \quad j \in \mathbb{Z},
$$

the building blocks in (4.3) have the form

$$
F_{\mu}:=P_{\eta, \beta} \varphi_{\eta} \varphi_{\theta}, \quad \mu=(\eta, \theta, \beta) \in \mathcal{M}_{j}
$$

where $P_{\eta, \beta}$ are defined in (3.8) and $\varphi_{\eta}, \varphi_{\theta}$ are from (3.4). We define

$$
\mathcal{F}_{j}:=\left\{F_{\mu}: \mu \in \mathcal{M}_{j}\right\} \quad \text { and } \quad W_{j}:=\operatorname{span} \mathcal{F}_{j}, \quad m \in \mathbb{Z} .
$$

To estimate the $L_{p}$-norms of the $F_{\mu}$ we shall make use of the following fact.

Lemma 4.1. The coefficients in (4.1) are uniformly bounded, that is,

$$
\left|m_{\alpha, \beta}^{\theta, \eta}\right| \leq c<\infty
$$

where $c$ depend only on $\mathbf{p}(\Theta), L$, and $k$.

Proof. Using (3.8) identity (4.1) can be rewritten as

$$
P_{\alpha} \circ A_{\theta}^{-1} \circ A_{\eta}=\sum_{|\beta|<k} m_{\alpha, \beta}^{\theta, \eta} P_{\beta},
$$

and since $\left\{P_{\beta}\right\}$ is an orthonormal basis for $\mathcal{P}_{k}$ in $L_{2}\left(B^{*}\right)$ we obtain

$$
\left|m_{\alpha, \beta}^{\theta, \eta}\right|=\left|\int_{B^{*}}\left(P_{\alpha} \circ A_{\theta}^{-1} \circ A_{\eta}\right)(y) P_{\beta}(y) d y\right| \leq c,
$$

where we have used condition (b) on $\Theta$ (see Definition 2.1).

Note that $\operatorname{supp} F_{\mu}=\overline{\theta \cap \eta}$ if $\mu=(\eta, \theta, \beta)$, and $\left\|F_{\mu}\right\|_{2} \sim 1$; hence $\left\|F_{\mu}\right\|_{\infty} \sim$ $|\theta \cap \eta|^{-1 / 2} \sim|\eta|^{-1 / 2}$ and

$$
\left\|F_{\mu}\right\|_{p} \sim|\eta|^{1 / p-1 / 2}, \quad 0<p \leq \infty .
$$

The main result of this section reads now as follows. 
Theorem 4.2. Any $f \in W_{j}$ has a unique representation

$$
f=\sum_{\mu \in \mathcal{M}_{j}} c_{\mu}(f) F_{\mu}
$$

where the dual functionals $c_{\mu}(\cdot)$ are of the following form: For each $\mu \in \mathcal{M}_{j}$, $\mu=(\eta, \theta, \beta)$, there is an ellipsoid $B_{\mu} \subset \theta \cap \eta$ with $\left|B_{\mu}\right| \sim|\eta|$ and $B_{\mu}=A_{\eta}\left(B_{\mu}^{*}\right)$ for some ball $B_{\mu}^{*} \subset B^{*}$ such that

$$
c_{\mu}(f)=\left\langle f, \tilde{F}_{\mu}\right\rangle, \quad \text { where } \operatorname{supp} \tilde{F}_{\mu} \subset \bar{B}_{\mu}, \quad\left\|\tilde{F}_{\mu}\right\|_{p} \sim|\eta|^{1 / p-1 / 2} .
$$

Moreover, if $f \in W_{j}$ and $f=\sum_{\mu \in \mathcal{M}_{j}} a_{\mu} F_{\mu}$, then

$$
\|f\|_{p} \sim\left(\sum_{\mu \in \mathcal{M}_{j}}\left\|a_{\mu} F_{\mu}\right\|_{p}^{p}\right)^{1 / p}, \quad 0<p \leq \infty
$$

with the obvious modification when $p=\infty$. Here all constants depend only on $\mathbf{p}(\Theta)$, $L$, and $k$.

The proof of this theorem is a mere repetition of the proof of Theorem 3.2 and is omitted. We observe, however, that the "coloring" scheme from $\S 3.1$ and the definition of the single level bases $\left\{\Phi_{m}\right\}$ in $\S 3.2$ were specifically designed to ensure the linear independence of the two-level-split bases.

We are now prepared to derive multilevel decompositions of functions using the two-level-split bases from above.

Theorem 4.3. For any $f \in L_{p}^{\text {loc }}$

$$
f=T_{0} f+\sum_{j \geq 0}\left(T_{j+1} f-T_{j} f\right)=\sum_{j \geq-1} \sum_{\mu \in \mathcal{M}_{j}} d_{\mu}(f) F_{\mu},
$$

where the convergence is in $L_{p}(K)$ for all compacta $K \subset \mathbb{R}^{n}$. Here for $j \geq 0$

$$
d_{\mu}(f)=b_{\eta, \beta}(f)-\sum_{|\alpha|<k} m_{\alpha, \beta}^{\theta, \eta} b_{\theta, \beta}(f), \quad \mu:=(\eta, \theta, \beta)
$$

with $m_{\alpha, \beta}^{\theta, \eta}$ from (4.1), while $\mathcal{M}_{-1}:=\Lambda_{0}, F_{\lambda}:=g_{\lambda}$, and $d_{\lambda}(f):=b_{\lambda}(f)$ if $\lambda \in \mathcal{M}_{-1}$. Moreover, if $f \in L_{p}\left(L_{\infty}:=C_{0}\right)$, then (4.11) as well as

$$
f=\sum_{j \in \mathbb{Z}}\left(T_{j+1}-T_{j}\right) f
$$

hold in $L_{p}$.

Proof. The relation (4.12 has been derived in (4.3) and the convergence of the lefthand side identity in (4.11) in $L_{p}(K)$ and $L_{p}$ follows from Lemma 3.3. To prove the convergence of (4.13) one has to show that $\left\|T_{j} f\right\|_{p} \rightarrow 0$ when $j \rightarrow-\infty$. Since this part of the claim will not be essential for the subsequent developments we only sketch the argument. Recall from Remark 7 in $\S 2.1$ that each $\theta \in \Theta_{j}$ expands to $\mathbb{R}^{n}$ as $j \rightarrow-\infty$. This implies that the basis elements $F_{\mu}, \mu \in \mathcal{M}_{j}$, become flatter and flatter as $j \rightarrow-\infty$, which easily leads to the desired convergence (see also the proof of Remark 5.1 below). 


\section{Anisotropic Besov spaces (B-spaces)}

In this section we introduce anisotropic Besov spaces induced by discrete ellipsoid covers of $\mathbb{R}^{n}$ and show that they can be characterized by the two-level-split bases from the previous section. Our approach to B-spaces is based on local polynomial approximation on the ellipsoids of the underlying discrete ellipsoid cover $\Theta$ of $\mathbb{R}^{n}$. Recall that the classical Besov spaces can also be defined via local polynomial approximation (local oscilations), see e.g. [19]. Furthermore, the anisotropic Besov space from $[14,10]$ are defined via local piecewise polynomial approximation. We maintain that local approximations rather than global means are more natural for the definition of anisotropic (and even classical) Besov spaces of positive smoothness since they more adequately reflect the nature of the spaces.

5.1. Homogeneous B-spaces. We now introduce the homogeneous B-spaces $\dot{B}_{p q}^{\alpha}(\Theta)$ induced by an arbitrary discrete ellipsoid cover $\Theta$ of $\mathbb{R}^{n}$. In the definition of the B-spaces $\dot{B}_{p q}^{\alpha}(\Theta)$ there is a hidden parameter $k$ which we choose to be the minimum integer satisfying the condition

$$
k>\frac{a_{0}}{a_{6}} \cdot \frac{\alpha}{n} .
$$

This will guarantee the equivalence of the norms in $\dot{B}_{p q}^{\alpha}(\Theta)$ introduced below. Here $a_{0}$ and $a_{6}$ are two of the parameters of $\Theta$ (see Definition 2.1). It can be shown that the norms in $\dot{B}_{p q}^{\alpha}(\Theta)$ with different $k$ 's satisfying (5.1) are equivalent.

Definition of $\dot{B}_{p q}^{\alpha}(\Theta)$ via local moduli of smoothness. For a given discrete ellipsoid cover $\Theta$ of $\mathbb{R}^{n}$ and $\alpha>0,0<p, q \leq \infty$, we define the space $\dot{B}_{p q}^{\alpha}(\Theta)$ as the set of all functions $f \in L_{p}^{\text {loc }}$ such that

$$
\|f\|_{\dot{B}_{p q}^{\alpha}(\Theta)}:=\left(\sum_{j \in \mathbb{Z}}\left(\sum_{\theta \in \Theta_{j}}|\theta|^{-\alpha p / n} \omega_{k}(f, \theta)_{p}^{p}\right)^{q / p}\right)^{1 / q}<\infty,
$$

where $\omega_{k}(f, \theta)_{p}$ is the $k$ th local modulus of smoothness of $f$ (see (3.20)).

As in (3.33) let $E_{k}(f, \theta)_{p}$ denote the best $L_{p}$ approximation of $f$ on $\theta$ from $\mathcal{P}_{k}$. By Whitney's theorem $E_{k}(f, \theta)_{p} \sim \omega_{k}(f, \theta)_{p}$ and hence replacing $\omega_{k}(f, \theta)_{p}$ in (5.2) by $E_{k}(f, \theta)_{p}$ will result in an equivalent norm.

The definition of $\dot{B}_{p q}^{\alpha}(\Theta)$ needs some additional clarification. Evidently the norm in $\dot{B}_{p q}^{\alpha}(\Theta)$ is not a true norm because $\|P\|_{\dot{B}_{p q}^{\alpha}(\Theta)}=0$ for $P \in \mathcal{P}_{k}$. Hence $\dot{B}_{p q}^{\alpha}(\Theta)$ is a quotient space modulo $\mathcal{P}_{k}$. We shall use the operators $Q_{j}, T_{j}$, and $T_{j, p}$ from $\S 3.3$ to construct a representer for each $f \in \dot{B}_{p q}^{\alpha}(\Theta)$. As before, let $T_{j}(j \in \mathbb{Z})$ be one of the operators $Q_{j}$ or $T_{j}$ or $T_{j, p}$, if $p \geq 1$, and $T_{j}:=T_{j, p}$, if $p<1$. We define

$$
\|f\|_{\dot{B}_{p q}^{\alpha}(\Theta)}^{T}:=\left(\sum_{j \in \mathbb{Z}}\left(2^{a_{0} j \alpha / n}\left\|\left(T_{j+1}-T_{j}\right) f\right\|_{p}\right)^{q}\right)^{1 / q} .
$$

On account of property (c) of ellipsoid covers Lemma 3.3 yields

$$
\left\|f-T_{j} f\right\|_{p} \leq c\left(\sum_{\theta \in \Theta_{j}} \omega_{k}(f, \theta)_{p}^{p}\right)^{1 / p}
$$

and using that $\left\|\left(T_{j+1}-T_{j}\right) f\right\|_{p} \leq c\left\|f-T_{j+1} f\right\|_{p}+c\left\|f-T_{j} f\right\|_{p}$, we obtain

$$
\|f\|_{\dot{B}_{p q}^{\alpha}(\Theta)}^{T} \leq c\|f\|_{\dot{B}_{p q}^{\alpha}(\Theta)}
$$


To describe the nature of $\dot{B}_{p q}^{\alpha}(\Theta)$ we have to distinguish two basic cases.

Case 1: $0<\alpha<n / p$ or $\alpha=n / p$ and $q \leq 1$.

We address first the scale components $\left(T_{j+1}-T_{j}\right) f$ for negative $j$.

Remark 5.1. Whenever $f \in \dot{B}_{p q}^{\alpha}(\Theta)$ for $0<\alpha<n / p$ or $\alpha=n / p$ and $q \leq 1$, there exists $P \in \mathcal{P}_{k}$ such that the series

$$
\sum_{j=-\infty}^{-1}\left(T_{j+1}-T_{j}\right) f=T_{0} f-P
$$

converges uniformly on $\mathbb{R}^{n}$.

Proof. Assume first that $0<\alpha<n / p$. By (5.4) it follows that

$$
\left\|\left(T_{j+1}-T_{j}\right) f\right\|_{p} \leq c 2^{-a_{0} j \alpha / n}\|f\|_{\dot{B}_{p q}^{\alpha}(\Theta)} .
$$

The function $\left(T_{j+1}-T_{j}\right) f$ is piecewise polynomial and hence for any $\eta \in \Theta_{j+1}$

$$
\begin{aligned}
\left\|\left(T_{j+1}-T_{j}\right) f\right\|_{L_{p}(\eta)} & \sim|\eta|^{1 / p}\left\|\left(T_{j+1}-T_{j}\right) f\right\|_{L_{\infty}(\eta)} \\
& \sim 2^{-a_{0} j / p}\left\|\left(T_{j+1}-T_{j}\right) f\right\|_{L_{\infty}(\eta)} .
\end{aligned}
$$

Therefore,

$$
\left\|\left(T_{j+1}-T_{j}\right) f\right\|_{\infty} \leq c 2^{a_{0} j(1 / p-\alpha / n)}\|f\|_{\dot{B}_{p q}^{\alpha}(\Theta)}
$$

which implies

$$
\sum_{j=-\infty}^{-1}\left\|\left(T_{j+1}-T_{j}\right) f\right\|_{\infty} \leq c\|f\|_{\dot{B}_{p q}^{\alpha}(\Theta)},
$$

since by assumption $\alpha<n / p$. Setting now $P:=T_{0} f-\sum_{j=-\infty}^{-1}\left(T_{j+1}-T_{j}\right) f$, and noting that each $T_{j} f$ belongs to $C\left(\mathbb{R}^{n}\right)$, we infer from (5.7) that $P \in C\left(\mathbb{R}^{n}\right)$ and

$$
T_{j} f \rightrightarrows P \quad \text { as } j \rightarrow-\infty \text {, uniformly on } \mathbb{R}^{n} \text {. }
$$

It is easy to see that $P \in \mathcal{P}_{k}$. Indeed, since the ellipsoids in $\Theta$ expand to $\mathbb{R}^{n}$ (see Remark 7 after Definition 2.1) any compact $K \subset \mathbb{R}^{n}$ is contained in a sequence $\left\{\theta_{\nu}^{\diamond}\right\}_{\nu=1}^{\infty}$ of ellipsoids dilated by a factor $a_{7}<1$ (see property (d) of $\Theta$ ) such that $\ell\left(\theta_{1}\right)>\ell\left(\theta_{2}\right)>\cdots$ and $\cup_{\nu \geq 0} \theta_{\nu}=\mathbb{R}^{n}$, where $\ell(\theta)=j$ means $\theta \in \Theta_{j}$. This implies $\varphi_{\theta_{\nu}} \rightrightarrows 1$ as $\nu \rightarrow \infty$ on $K$. Now, taking into account the definition of $T_{j}$ this leads to $T_{j} f \rightrightarrows P_{K}$ as $j \rightarrow-\infty$ for some $P_{K} \in \mathcal{P}_{k}$ on all compact sets $K$. Therefore, (5.8) holds for some $P \in \mathcal{P}_{k}$ and (5.5) follows.

If $\alpha=n / p$ and $q \leq 1$, we argue in a similar fashion that

$$
\begin{aligned}
\left(\sum_{j=-\infty}^{-1}\left\|\left(T_{j+1}-T_{j}\right) f\right\|_{\infty}\right)^{q} & \leq \sum_{j=-\infty}^{-1}\left\|\left(T_{j+1}-T_{j}\right) f\right\|_{\infty}^{q} \\
& \left.\leq \sum_{j=-\infty}^{-1} 2^{a_{0} j q \alpha / n}\left\|\left(T_{j+1}-T_{j}\right) f\right\|_{p}^{q} \leq c\|f\|_{\dot{B}_{p q}^{\alpha}(\Theta)}^{q}\right)
\end{aligned}
$$

which also yields (5.7) and allows us to continue as before.

We turn now to the series $\sum_{j=0}^{\infty}\left(T_{j+1}-T_{j}\right) f$. 
Remark 5.2. If $f \in \dot{B}_{p q}^{\alpha}(\Theta), \alpha>0,0<p, q \leq \infty$, then

$$
\sum_{j=0}^{\infty}\left(T_{j+1}-T_{j}\right) f=f-T_{0} f \quad \text { in } \quad L_{p} .
$$

Proof. Let first $p \geq 1$. Since by (5.4)

$$
\sum_{j=0}^{\infty}\left(2^{a_{0 j \alpha} / n}\left\|\left(T_{j+1}-T_{j}\right) f\right\|_{p}\right)^{q} \leq c\|f\|_{\dot{B}_{p q}^{\alpha}}^{q},
$$

we have $\left\|\left(T_{j+1}-T_{j}\right) f\right\|_{p} \leq c 2^{-a_{0} j \alpha / n}\|f\|_{\dot{B}_{p q}^{\alpha}}$ and therefore

$$
\sum_{j=0}^{\infty}\left\|\left(T_{j+1}-T_{j}\right) f\right\|_{p} \leq c\|f\|_{\dot{B}_{p q}^{\alpha}} .
$$

One readily infers from (5.11) that the partial sums $\sum_{j=0}^{J}\left(T_{j+1}-T_{j}\right) f$ form a Cauchy sequence in $L_{p}$. By Lemma 3.3 the limit must agree with $f-T_{0} f$, which confirms (5.10) for $p \geq 1$.

For $p<1$ we shall show first that

$$
\sum_{j=0}^{\infty}\left\|\left(T_{j+1}-T_{j}\right) f\right\|_{p}^{p} \leq c\|f\|_{\dot{B}_{p q}^{\alpha}}^{p} .
$$

In fact, when $q \leq p$ we deduce from (5.10) that

$$
\sum_{j=0}^{\infty} 2^{a_{0} j \alpha / n}\left\|\left(T_{j+1}-T_{j}\right) f\right\|_{p} \leq c\|f\|_{\dot{B}_{p q}^{\alpha}}
$$

and therefore $\left\|\left(T_{j+1}-T_{j}\right) f\right\|_{p}^{p} \leq c 2^{-a_{0} j p \alpha / n}\|f\|_{\dot{B}_{p q}^{\alpha}}^{p}$ which yields (5.12) for $q \leq p$. When $q>p$ we apply Hölder's inequality to

$$
\sum_{j=0}^{\infty}\left\|\left(T_{j+1}-T_{j}\right) f\right\|_{p}^{p}=\sum_{j=0}^{\infty} 2^{-a_{0} j \alpha p / n}\left(2^{a_{0} j \alpha / n}\left\|\left(T_{j+1}-T_{j}\right) f\right\|_{p}\right)^{p}
$$

with $\tilde{p}:=q / p>1$ to conclude (5.12) in this case as well. Now since

$$
\left\|\sum_{j=J}^{J+l}\left(T_{j+1}-T_{j}\right) f\right\|_{p}^{p} \leq \sum_{j=J}^{J+l}\left\|\left(T_{j+1}-T_{j}\right) f\right\|_{p}^{p} \rightarrow 0 \quad \text { as } J \rightarrow \infty
$$

and using again that $T_{j} f \rightarrow f$ in $L_{p}(K)$ on each compact $K \subset \mathbb{R}^{n}$ (see Lemma 3.3) we arrive at (5.9) also in the case $p<1$.

Remarks 5.1-5.2 reveal the structure of the functions in $\dot{B}_{p q}^{\alpha}(\Theta)$ under the above circumstances.

Remark 5.3. Let $0<\alpha<n / p$ or $\alpha=n / p$ and $q \leq 1$. Then for any $f \in \dot{B}_{p q}^{\alpha}(\Theta)$ there exists $P \in \mathcal{P}_{k}$ such that $f=\sum_{j \in \mathbb{Z}}\left(T_{j+1}-T_{j}\right) f+P$ in $L_{p}(K)$ for all compact sets $K \subset \mathbb{R}^{n}$. Thus, one can define $\dot{B}_{p q}^{\alpha}(\Theta)$, in this case, as the set of all functions $f \in L_{p}^{\text {loc }}$ such that $\|f\|_{\dot{B}_{p q}^{\alpha}}<\infty$ and

$$
f=\sum_{j \in \mathbb{Z}}\left(T_{j+1}-T_{j}\right) f \quad \text { in } \quad L_{p}(K) \quad \text { for all compact sets } K \subset \mathbb{R}^{n} .
$$


In addition, by (5.7) and (5.12) it follows that

$$
\|f\|_{L_{p}(K)} \leq c\left(1+|K|^{1 / p}\right)\|f\|_{\dot{B}_{p q}^{\alpha}} \text { for all compact sets } K \subset \mathbb{R}^{n} .
$$

In order to refine the above observations we consider the following two subcases corresponding to lower, respectively higher smoothness $\alpha$.

Case 1 (a): $0<\alpha<n(1 / p-1)$ or $0<\alpha=n(1 / p-1)$ and $q>1$.

In this case we necessarily have $0<p<1$. Moreover, if we were to deal with the classical Besov spaces, the above condition on $\alpha$ means that embedding in $\mathcal{S}^{\prime}$ fails. Thus, we are content with the convergence from above in this case.

Alternatively we have

Case 1 (b): $n(1 / p-1)<\alpha<n / p$ or $\alpha=n(1 / p-1)$ and $q \leq 1$ or $\alpha=n / p$ and $q \leq 1$.

For classical Besov spaces this is known to imply that $\dot{B}_{p q}^{\alpha}$ is embedded in $\mathcal{S}^{\prime}$ which persists to hold here as well, as will be shown next. The main observation can be stated as follows.

Remark 5.4. Let $\alpha>n(1 / p-1)$ or $\alpha=n(1 / p-1)$ and $q \leq 1$. Then for any $f \in \dot{B}_{p q}^{\alpha}(\Theta)$

$$
\sum_{j=0}^{\infty}\left(T_{j+1}-T_{j}\right) f=f-T_{0} f \quad \text { in } \quad \mathcal{S}^{\prime} .
$$

Proof. From Remark 5.2 we already know that $\sum_{j=0}^{\infty}\left(T_{j+1}-T_{j}\right) f$ converges in $L_{p}$ and thus in $\mathcal{S}^{\prime}$ whenever $p \geq 1$, i.e. (5.15) holds for $p \geq 1$.

Suppose now that $p<1$. Since $\left(T_{j+1}-T_{j}\right) f$ is a piecewise polynomial, then we have, using the notation of Theorem 4.3,

$$
\begin{aligned}
\left\|\left(T_{j+1}-T_{j}\right) f\right\|_{1} & \leq \sum_{\mu \in \mathcal{M}_{j}}\left\|d_{\mu} F_{\mu}\right\|_{1} \leq c \sum_{\mu \in \mathcal{M}_{j}}\left|\eta_{\mu}\right|^{1-1 / p}\left\|d_{\mu} F_{\mu}\right\|_{p} \\
& \leq c 2^{-a_{0} j(1-1 / p)}\left(\sum_{\mu \in \mathcal{M}_{j}}\left\|d_{\mu} F_{\mu}\right\|_{p}^{p}\right)^{1 / p} \\
& \leq c 2^{-a_{0} j(1-1 / p)}\left\|\left(T_{j+1}-T_{j}\right) f\right\|_{p} .
\end{aligned}
$$

Thus, on account of (5.10), we obtain

$$
\sum_{j=0}^{\infty}\left(2^{a_{0} j[\alpha / n-(1 / p-1)]}\left\|\left(T_{j+1}-T_{j}\right) f\right\|_{p}\right)^{q} \leq c\|f\|_{\dot{B}_{p q}^{\alpha}}^{q},
$$

which implies

$$
\sum_{j=0}^{\infty}\left\|\left(T_{j+1}-T_{j}\right) f\right\|_{1} \leq c\|f\|_{\dot{B}_{p q}^{\alpha}} .
$$

By the argument in the proof of Remark 5.2, see (5.11), we conclude that

$$
\sum_{j=0}^{\infty}\left(T_{j+1}-T_{j}\right) f=f-T_{0} f \quad \text { in } \quad L_{1} \quad \text { and hence in } \mathcal{S}^{\prime},
$$

which completes the proof.

In summary, from Remark 5.1 and 5.4 we can state the following. 
Remark 5.5. Let $\alpha>0$ and suppose $n(1 / p-1)<\alpha<n / p$ or $\alpha=n(1 / p-1)$ and $q \leq 1$ or $\alpha=n / p$ and $q \leq 1$. Then, for any $f \in \dot{B}_{p q}^{\alpha}(\Theta)$ there exists $P \in \mathcal{P}_{k}$ such that $f=\sum_{j \in \mathbb{Z}}\left(T_{j+1}-T_{j}\right) f+P$ converges in $\mathcal{S}^{\prime}$. Thus, the space $\dot{B}_{p q}^{\alpha}(\Theta)$ can be defined as the set of all regular tempered distributions $f$ such that $\|f\|_{\dot{B}_{p q}^{\alpha}(\Theta)}<\infty$ and

$$
f=\sum_{j \in \mathbb{Z}}\left(T_{j+1}-T_{j}\right) f \quad \text { in } \quad \mathcal{S}^{\prime}
$$

Also, it follows from above that

$$
|\langle f, \phi\rangle| \leq c\|f\|_{\dot{B}_{p q}^{\alpha}(\Theta)}\|\phi\|_{\infty}, \quad \forall \phi \in \mathcal{S},
$$

i.e. $\dot{B}_{p q}^{\alpha}(\Theta)$ is continuously embedded in $\mathcal{S}^{\prime}$.

We now turn to

Case 2: $\alpha>n / p$ or $\alpha=n / p$ and $q>1$.

As in Case $1(\mathrm{~b})$ since $\alpha>n(1 / p-1)$, we have

$$
\sum_{j=0}^{\infty}\left(T_{j+1}-T_{j}\right) f=f-T_{0} f \quad \text { in } \quad \mathcal{S}^{\prime} .
$$

But now, dealing with the series $\sum_{j=-\infty}^{-1}\left(T_{j+1}-T_{j}\right) f$ is more complicated because it is not convergent. As in the classical case we need to renormalize this series.

Lemma 5.6. There exist polynomials $P \in \mathcal{P}_{k}$ and $P_{j} \in \mathcal{P}_{k}, j=-1,-2, \ldots$, such that

$$
T_{0} f=P+\lim _{m \rightarrow-\infty} \sum_{j=m}^{-1}\left(T_{j+1}-T_{j}\right) f-P_{j} \quad \text { in } \quad \mathcal{S}^{\prime}
$$

and also uniformly on every compact set $K \subset \mathbb{R}^{n}$. Moreover, if $R_{j}$ is the $k-1$ degree Taylor polynomial of the function $\left(T_{j+1}-T_{j}\right) f \circ A_{\theta}^{-1}$ based at 0 for some $\theta \in \Theta_{0}$, then $P_{j}$ can be defined as $P_{j}:=R_{j} \circ A_{\theta}$.

Proof. From (5.4) it follows that

$$
\sum_{j=-\infty}^{-1}\left(2^{a_{0} j \alpha / n}\left\|\left(T_{j+1}-T_{j}\right) f\right\|_{p}\right)^{q} \leq c\|f\|_{\dot{B}_{p q}^{\alpha}(\Theta)}^{q}=: \mathcal{N}^{q} .
$$

Hence

and by $(5.6)$

$$
\left\|\left(T_{j+1}-T_{j}\right) f\right\|_{p} \leq 2^{-a_{0} j \alpha / n} \mathcal{N}
$$

Note that $\|f\|_{\dot{B}_{p q}^{\alpha}(\Theta)}$ does not change when applying to $f$ and the ellipsoids in $\Theta$ an affine transform $A(x)=M x+v$ with $|\operatorname{det} M|=1$. Also, $A$ is a homeomorphism from $\mathcal{S}$ onto itself. Therefore, we may assume that $B(0,1) \in \Theta_{0}$.

Let $P_{j}$ be the $k-1$ degree Taylor polynomial of $\left(T_{j+1}-T_{j}\right) f$ based at $x=0$. We need to estimate $\left|\left(T_{j+1}-T_{j}\right) f(x)-P_{j}(x)\right|$ for all $x \in \mathbb{R}^{n}$. To this end we next estimate $\left|\partial^{\beta}\left(T_{j+1}-T_{j}\right) f(x)\right|$ whenever $|\beta|=k$. 
Let first $\theta \in \Theta_{j}, j<0$, be such that $0 \in \theta$. By the definition of $T_{j}$, the properties of $\Theta$, and (4.10) (with $p=\infty$ ) it follows that

$\left\|\partial^{\beta}\left(T_{j+1}-T_{j}\right) f \circ A_{\theta}\right\|_{L_{\infty}\left(B^{*}\right)} \leq c\left\|\left(T_{j+1}-T_{j}\right) f \circ A_{\theta}\right\|_{L_{\infty}\left(B^{*}\right)}=c\left\|\left(T_{j+1}-T_{j}\right) f\right\|_{L_{\infty}(\theta)}$,

which leads to

$$
\begin{aligned}
\left\|\partial^{\beta}\left(T_{j+1}-T_{j}\right) f\right\|_{L_{\infty}(\theta)} & \leq\left(\left\|A_{\theta}^{-1}\right\|_{\ell_{2} \rightarrow \ell_{2}}\right)^{|\beta|}\left\|\partial^{\beta}\left(T_{j+1}-T_{j}\right) f \circ A_{\theta}\right\|_{L_{\infty}\left(B^{*}\right)} \\
& \leq c\left[\sigma_{\min }(\theta)\right]^{-k}\left\|\left(T_{j+1}-T_{j}\right) f\right\|_{L_{\infty}(\theta)} \\
& \leq c 2^{a_{6} j k} 2^{-a_{0} j(\alpha / n-1 / p)} \mathcal{N}=c 2^{j \varepsilon} \mathcal{N}
\end{aligned}
$$

where $\varepsilon:=a_{6}\left(k-\frac{a_{0}}{a_{6}} \cdot \frac{\alpha}{n}+\frac{a_{0}}{a_{6} p}\right)$ and $\varepsilon>0$ by (5.1). Here, we have used Lemma 2.2 for the estimate $\sigma_{\min }(\theta) \geq\left(1 / a_{5}\right) 2^{-a_{6} j} \sigma_{\min }\left(B^{*}\right)=\left(1 / a_{5}\right) 2^{-a_{6} j}$.

Now, suppose $|x|>1$ and let $x \in \eta, \eta \in \Theta_{j}$. Let $\theta^{\prime} \in \Theta$ be an ellipsoid of maximum level such that $0, x \in \theta^{\prime}$. Then $\theta^{\prime} \in \Theta_{j-r}$ for some $r \geq 0$. By Lemma 2.2 $\sigma_{\min }\left(\theta^{\prime}\right) \geq\left(1 / a_{5}\right) 2^{-a_{6}(j-r)}$ and since $\eta \cap \theta^{\prime} \neq \emptyset$ from this and again Lemma 2.2 it follows that

$$
\sigma_{\min }(\eta) \geq a_{3} 2^{-a_{4} \nu} \sigma_{\min }\left(\theta^{\prime}\right) \geq c 2^{-a_{6}(j-r)} 2^{-a_{4} r}=c 2^{-\left(a_{4}-a_{6}\right) r} 2^{-a_{6} j} .
$$

Now, as above we infer

$$
\begin{aligned}
\left\|\partial^{\beta}\left(T_{j+1}-T_{j}\right) f\right\|_{L_{\infty}(\eta)} & \leq c\left[\sigma_{\min }(\eta)\right]^{-k}\left\|\left(T_{j+1}-T_{j}\right) f\right\|_{L_{\infty}(\eta)} \\
& \leq c 2^{\left(a_{4}-a_{6}\right) r k} 2^{a_{6} j k} 2^{-a_{0} j(\alpha / n-1 / p)} \mathcal{N}=c 2^{\left(a_{4}-a_{6}\right) r k} 2^{j \varepsilon} \mathcal{N} .
\end{aligned}
$$

We need a lower bound for $|x|$ in terms of $j$ and $r$. By property (d) of $\Theta$ there exists an ellipsoid $\theta^{\prime \prime} \in \Theta_{j-r+1}$ such that $0 \in \theta^{\prime \prime \diamond}$, where $\theta^{\prime \prime \diamond}$ is the dilated version of $\theta^{\prime \prime}$ by a factor of $a_{7}<1$. Since $\theta^{\prime}$ was selected of minimum level so that $0, x \in \theta^{\prime}$, then $x \notin \theta^{\prime \prime}$ and hence $|x| \geq\left(1-a_{7}\right) \sigma_{\min }\left(\theta^{\prime \prime}\right)$. On the other hand, by Lemma 2.2

$$
\sigma_{\min }\left(\theta^{\prime \prime}\right) \geq c \sigma_{\min }\left(\theta^{\prime}\right) \geq c 2^{-a_{6}(j-r)} .
$$

Hence, $|x| \geq c 2^{-a_{6}(j-r)} \geq c 2^{a_{6} r}$. Combining this with (5.23) and (5.22), we get

$$
\left|\partial^{\beta}\left(T_{j+1}-T_{j}\right) f(x)\right| \leq c(|x|+1)^{\gamma} 2^{j \varepsilon} \mathcal{N}, \quad x \in \mathbb{R}^{n},
$$

where $\gamma:=\frac{a_{4}-a_{6}}{a_{6}} k$. Now, Taylor's theorem gives

$$
\begin{aligned}
\left|\left(T_{j+1}-T_{j}\right) f(x)-P_{j}(x)\right| & \leq c \sum_{|\beta|=k}\left\|\partial^{\beta}\left(T_{j+1}-T_{j}\right) f\right\|_{L_{\infty}(B(0,|x|)}|x|^{k} \\
& \leq c(|x|+1)^{\gamma+k} 2^{j \varepsilon} \mathcal{N} .
\end{aligned}
$$

Therefore, for any $R>0$ we conclude that

$$
\sum_{j=-\infty}^{-1}\left\|\left(T_{j+1}-T_{j}\right) f-P_{j}\right\|_{L_{\infty}(B(0, R))}<\infty .
$$

Setting

$$
P:=T_{0} f-\sum_{j=-\infty}^{-1}\left(T_{j+1}-T_{j}\right) f-P_{j} \quad \text { and } \quad Q_{j}:=\sum_{\nu=j}^{-1} P_{\nu}, \quad Q_{j} \in \mathcal{P}_{k},
$$

we evidently have $P \in C\left(\mathbb{R}^{n}\right)$. Then by (5.25) $T_{j} f+Q_{j} \rightrightarrows P$ as $j \rightarrow-\infty$ uniformly on $B(0, R)$ for all $R>0$. As in Case 1 one easily shows that $P \in \mathcal{P}_{k}$. 
We now employ (5.24) to find that for any $\phi \in \mathcal{S}$

$$
\begin{aligned}
\left|\left\langle\sum_{j=-\infty}^{m}\left(T_{j+1}-T_{j}\right) f-P_{j}, \phi\right\rangle\right| & \leq c \sum_{j=-\infty}^{m} 2^{j \varepsilon} \mathcal{N}\left|\left\langle(|\cdot|+1)^{\gamma+k}, \phi\right\rangle\right| \\
& \leq c 2^{m \varepsilon} \mathcal{N}\|\phi\|_{\gamma+k} \rightarrow 0, \quad m \rightarrow-\infty
\end{aligned}
$$

where $\|\phi\|_{s}:=\left\|(|x|+1)^{s} \phi(x)\right\|_{\infty}$. Hence

$$
\sum_{j=-\infty}^{-1}\left(T_{j+1}-T_{j}\right) f-P_{j}=T_{0} f-P \quad \text { in } \quad \mathcal{S}^{\prime} \quad\left(P \in \mathcal{P}_{k}\right)
$$

which completes the proof of Lemma 5.6.

In summary, from (5.18) and Lemma 5.6 we conclude the following.

Remark 5.7. Whenever $\alpha>n / p$ or $\alpha=n / p$ and $q>1$ the space $\dot{B}_{p q}^{\alpha}(\Theta)$ can be viewed as the set of all regular tempered distributions $f$ such that $\|f\|_{\dot{B}_{p q}^{\alpha}(\Theta)}<\infty$ and

$$
f=\sum_{j \in \mathbb{Z}}\left(T_{j+1}-T_{j}\right) f
$$

where the convergence is in $S^{\prime} / \mathcal{P}_{k}$. This means that there exist polynomials $P \in \mathcal{P}_{k}$ and $P_{j} \in \mathcal{P}_{k}, j \in \mathbb{Z}$, such that

$$
f=P+\lim _{m \rightarrow-\infty} \sum_{j=m}^{\infty}\left(T_{j+1}-T_{j}\right) f+P_{m} \quad \text { in } \quad \mathcal{S}^{\prime} .
$$

Moreover, $\dot{B}_{p q}^{\alpha}(\Theta)$ can be defined as the set of all $f \in \mathcal{S}^{\prime}$ such that $\|f\|_{\dot{B}_{p q}^{\alpha}(\Theta)}<\infty$ and

$$
f=\sum_{j \in \mathbb{Z}}\left(T_{j+1}-T_{j}\right) f-P_{j} \quad \text { in } \quad \mathcal{S}^{\prime}
$$

where the polynomials $P_{j}$ are defined as in Lemma 5.6 for $j \leq-1$ and $P_{j}:=0$ if $j \geq 0$. In addition, $\dot{B}_{p q}^{\alpha}(\Theta)$ is continuously embedded in $\mathcal{S}^{\prime}$, namely, for $f \in \dot{B}_{p q}^{\alpha}(\Theta)$

$$
|\langle f, \phi\rangle| \leq c\|f\|_{\dot{B}_{p q}^{\alpha}(\Theta)}\|\phi\|_{\gamma+k}, \quad \forall \phi \in \mathcal{S} .
$$

This last estimate follows from (5.18) and (5.26).

Other norms in $\dot{B}_{p q}^{\alpha}(\Theta)$. The understanding of the B-spaces relies to a great extent on having several equivalent norms for $\dot{B}_{p q}^{\alpha}(\Theta)$ at hand, as will be exemplified later for best $m$-term approximation with the two-level-split basis functions. Observe first that if

$$
\left(T_{j+1}-T_{j}\right) f=\sum_{\mu \in \mathcal{M}_{j}} d_{\mu}(f) F_{\mu},
$$

then, using Theorem 4.2, we can write

$$
\|f\|_{\dot{B}_{p q}^{\alpha}(\Theta)}^{T} \sim\left(\sum_{j \in \mathbb{Z}}\left(\sum_{\mu \in \mathcal{M}_{j}}\left(\left|\eta_{\mu}\right|^{-\alpha / n}\left\|d_{\mu}(f) F_{\mu}\right\|_{p}\right)^{p}\right)^{q / p}\right)^{1 / q} .
$$

We also define

$$
\|f\|_{\dot{B}_{p q}^{\alpha}(\Theta)}^{A}:=\inf _{f=\sum_{\mu \in \mathcal{M}} a_{\mu} F_{\mu}}\left(\sum_{j \in \mathbb{Z}}\left(\sum_{\mu \in \mathcal{M}_{j}}\left(\left|\eta_{\mu}\right|^{-\alpha / n}\left\|a_{\mu} F_{\mu}\right\|_{p}\right)^{p}\right)^{q / p}\right)^{1 / q} .
$$


Here the infimum is taken over all representations $f=\sum_{\mu \in \mathcal{M}} a_{\mu} F_{\mu}$, where the convergence is to be understood as described above.

Central for this part is the equivalence of the above norms.

Theorem 5.8. If $\alpha>0,0<p, q \leq \infty$, and (5.1) is obeyed, then the norms $\|\cdot\|_{\dot{B}_{p q}^{\alpha}(\Theta)},\|\cdot\|_{\dot{B}_{p q}^{\alpha}(\Theta)}^{T}$, and $\|\cdot\|_{\dot{B}_{p q}^{\alpha}(\Theta)}^{A}$ are equivalent.

We defer the somewhat lengthy proof of this theorem to the appendix.

One uses in a standard way the embedding of $\dot{B}_{p q}^{\alpha}$ in $\mathcal{S}^{\prime}$ or (5.14) to show its completeness. We record this in the following proposition.

Proposition 5.9. For $0<p, q \leq \infty$ and $\alpha>0$ the B-space $\dot{B}_{p q}^{\alpha}$ is a quasi-Banach space which is continuously embedded in $\mathcal{S}^{\prime}$ if $\alpha>n(1 / p-1)$ or $\alpha=n(1 / p-1)$ and $q \leq 1$.

5.2. Inhomogeneous B-spaces. Sometimes it is more convenient to use the inhomogeneous version $B_{p q}^{\alpha}\left(\Theta^{+}\right)$of the B-spaces induced by anisotropic ellipsoid covers of $\mathbb{R}^{n}$. For the inhomogeneous B-spaces we only need ellipsoid covers with levels $j=0,1, \ldots$, i.e. covers of the form

$$
\Theta^{+}:=\bigcup_{j=0}^{\infty} \Theta_{j} .
$$

In a certain sense the spaces $B_{p q}^{\alpha}\left(\Theta^{+}\right)$are simpler than the homogeneous counterparts $\dot{B}_{p q}^{\alpha}(\Theta)$ which justifies a fairly brief exposition.

As for the homogeneous $\mathrm{B}$-spaces there is a hidden index $k$ which is also present in the definition of basis elements. It will always be selected to be the minimum integer such that

$$
k>\frac{a_{0}}{a_{6}} \cdot \frac{\alpha}{n},
$$

where $a_{0}, a_{6}>0$ are from the definition of discrete ellipsoid covers in $\S 2.1$.

Definition of $B_{p q}^{\alpha}\left(\Theta^{+}\right)$via local moduli of smoothness. For given discrete ellipsoid cover $\Theta^{+}$of $\mathbb{R}^{n}$ and $\alpha>0,0<p, q \leq \infty$, we define the space $B_{p q}^{\alpha}\left(\Theta^{+}\right)$as the set of all functions $f \in L_{p}$ such that

$$
|f|_{B_{p q}^{\alpha}\left(\Theta^{+}\right)}:=\left(\sum_{j \geq 0}\left(\sum_{\theta \in \Theta_{j}}\left(|\theta|^{-\alpha p / n} \omega_{k}(f, \theta)_{p}^{p}\right)^{q / p}\right)^{1 / q}<\infty,\right.
$$

where $\omega_{k}(f, \theta)_{p}$ is the $k$ th local modulus of smoothness of $f$ in $L_{p}(\theta)$. The norm in $B_{p q}^{\alpha}$ is defined by

$$
\|f\|_{B_{p q}^{\alpha}\left(\Theta^{+}\right)}:=\|f\|_{p}+|f|_{B_{p q}^{\alpha}\left(\Theta^{+}\right)} .
$$

Other norms on $\boldsymbol{B}_{\boldsymbol{p q}}^{\boldsymbol{\alpha}}\left(\boldsymbol{\Theta}^{+}\right)$. As in the homogeneous case, let $T_{j}(j \in \mathbb{Z})$ be one of the operators $Q_{j}$ or $T_{j}$ or $T_{j, p}$, if $p \geq 1$, and $T_{j}:=T_{j, p}$, if $p<1$, defined in $\S 3.3$. In view of the representation of functions $f \in L_{p}$ from Theorem 4.3, we define

$$
\|f\|_{B_{p q}^{\alpha}\left(\Theta^{+}\right)}^{T}:=\left\|T_{0} f\right\|_{p}+\left(\sum_{j \geq 0}\left(2^{a_{0} j \alpha / n}\left\|\left(T_{j+1}-T_{j}\right) f\right\|_{p}\right)^{q}\right)^{1 / q} .
$$


By Theorem 4.2 and (4.10)

$$
\|f\|_{B_{p q}^{\alpha}\left(\Theta^{+}\right)}^{T} \sim\left(\sum_{j \geq-1}\left(\sum_{\mu \in \mathcal{M}_{j}}\left(\left|\eta_{\mu}\right|^{-\alpha / n}\left\|d_{\mu}(f) F_{\mu}\right\|_{p}\right)^{p}\right)^{q / p}\right)^{1 / q} .
$$

Here $\mathcal{M}_{-1}:=\Lambda_{0}, F_{\lambda}:=g_{\lambda}$, and $d_{\lambda}(f):=b_{\lambda}(f)$ if $\lambda \in \mathcal{M}_{-1}$.

Finally, we define again yet another norm on $B_{p q}^{\alpha}\left(\Theta^{+}\right)$via basis elements:

$$
\|f\|_{B_{p q}^{\alpha}\left(\Theta^{+}\right)}^{A}:=\inf _{f=\sum_{\mu \in \mathcal{M}^{+}} a_{\mu} F_{\mu}}\left(\sum_{j \geq-1}\left(\sum_{\mu \in \mathcal{M}_{j}}\left(\left|\eta_{\mu}\right|^{-\alpha / n}\left\|a_{\mu} F_{\mu}\right\|_{p}\right)^{p}\right)^{q / p}\right)^{1 / q}
$$

where the infimum is taken over all representations $f=\sum_{\mu \in \mathcal{M}^{+}} a_{\mu} F_{\mu}$ in $L_{p}$ with $\mathcal{M}^{+}:=\cup_{j \geq-1} \mathcal{M}_{j}$.

It is important that the above norms are equivalent.

Theorem 5.10. Suppose $\alpha>0,0<p, q \leq \infty$, and (5.1) holds. Then the norms $\|\cdot\|_{B_{p q}^{\alpha}\left(\Theta^{+}\right)},\|\cdot\|_{B_{p q}^{\alpha}\left(\Theta^{+}\right)}^{T}$, and $\|\cdot\|_{B_{p q}^{\alpha}\left(\Theta^{+}\right)}^{A}$ are equivalent.

The proof of this theorem is quite similar to the proof of Theorem 5.8 and will be omitted.

Similarly as $\dot{B}_{p q}^{\alpha}(\Theta)$ the inhomogeneous B-space $B_{p q}^{\alpha}\left(\Theta^{+}\right)$is a quasi-Banach space. We omit the details.

Remark 5.11. There are continuous and semi-continuous counterparts of the $B$ spaces $\dot{B}_{p q}^{\alpha}(\Theta)$ and $B_{p q}^{\alpha}\left(\Theta^{+}\right)$defined in this section. For instance, the B-space $\dot{B}_{p q}^{\alpha}(\Theta)$ can be defined for any continuous ellipsoid cover $\Theta$ of $\mathbb{R}^{n}$ as the set of all functions $f \in L_{p}^{\text {loc }}$ such that

$$
\|f\|_{\dot{B}_{p q}^{\alpha}(\Theta)}:=\left(\int_{0}^{\infty}\left(t^{\alpha / d+1} \int_{\mathbb{R}^{n}} \omega_{k}(f, \theta(v, t))_{p}^{p} d v\right)^{q / p} d t / t\right)^{1 / q}<\infty .
$$

Here one should assume that $|\theta(v, t)|$ is a measurable function of $v, t$. We omit the details.

5.3. Interrelation of B-spaces with Besov and other B-spaces. It is quite easy to show that if $\Theta$ is a regular ellipsoid (ball) cover of $\mathbb{R}^{n}$, then the B-spaces $\dot{B}_{p q}^{\alpha}(\Theta)$ and $B_{p q}^{\alpha}\left(\Theta^{+}\right)$considered in this paper are the same as the corresponding classical Besov spaces (see also [9]).

As already mentioned the powers $A^{j}$ of a real $n \times n$ matrix $A$ with eigenvalues $\lambda$ obeying $|\lambda|>1$ generate a semi-continuous and hence discrete ellipsoid cover of $\mathbb{R}^{n}$. It can be shown that for $\alpha>n(1 / p-1)_{+}$the associated B-space $\dot{B}_{p q}^{\alpha}$ are exactly the same (with equivalent norms) as the anisotropic Besov spaces (with weight 1 ) developed in [2].

The B-spaces from this paper have a lot in common with the B-spaces introduced in $[14,10]$. The main distinctions are that the latter B-spaces are based on multilevel nested triangulations of $\mathbb{R}^{2}$ and local piecewise polynomial approximation while the former are based on ellipsoid covers and local polynomial approximation. Consequently, the B-norms based on nested triangulations can be in general much smaller than the B-norms from this paper.

As indicated in Proposition 2.10, $\mathbb{R}^{n}$ equipped with the distance introduced in Definition 2.6 and the Lebesgue measure is a space of homogeneous type and hence the general theory of Besov spaces on homogeneous spaces applies (see e.g. [13]). 
In fact, in the specific setting of this paper the anisotropic Besov spaces given by the general theory are the same as the B-spaces from here for sufficiently small $\alpha>0$. The main distinction between the two theories is that we can handle Bspaces of an arbitrary smoothness $\alpha>0$, while the general theory of Besov spaces on homogeneous spaces is only feasible for smoothness $\alpha$ with $|\alpha|<\varepsilon$ for some sufficiently small $\varepsilon$.

\section{NonLinear APPROXimation}

In this section, we apply the B-spaces to nonlinear $m$-term approximation from the two-level-split bases introduced in $\S 4$.

6.1. The B-spaces of nonlinear approximation. A specific type of B-spaces will play an important role in nonlinear m-term approximation in $L_{p}$. For $0<p<$ $\infty$ and $\alpha>0$ let $\tau$ be determined by

$$
1 / \tau=\alpha / n+1 / p
$$

which in the case of classical Besov spaces signifies the critical embedding in $L_{p}$. For nonlinear approximation in $L_{\infty}:=C_{0}(p=\infty) \tau$ is determined by $1 / \tau=\alpha / n$ and we shall necessarily assume that $\alpha \geq 1$ (otherwise the embedding (6.5) below is not valid).

For a given discrete ellipsoid cover $\Theta$ of $\mathbb{R}^{n}$, consider the homogeneous B-space $\dot{B}_{\tau}^{\alpha}:=\dot{B}_{\tau \tau}^{\alpha}(\Theta)$. Then

$$
\|f\|_{\dot{B}_{\tau}^{\alpha}(\Theta)}:=\left(\sum_{\theta \in \Theta}|\theta|^{-\alpha \tau / n} \omega_{k}(f, \theta)_{\tau}^{\tau}\right)^{1 / \tau} .
$$

Although in general $\tau<1$, it can be proved (similarly as in [14]) that for any $0<q<p$

$$
\|f\|_{\dot{B}_{\tau}^{\alpha}(\Theta)} \sim\left(\sum_{\theta \in \Theta}|\theta|^{(1 / p-1 / q) \tau} \omega_{k}(f, \theta)_{q}^{\tau}\right)^{1 / \tau},
$$

which allows one to work in $L_{q}$ with $q \geq 1$ if $p>1$.

From (5.27) and (4.7) one readily derives this representation of the norm in $\dot{B}_{\tau}^{\alpha}(\Theta)$ :

$$
\|f\|_{\dot{B}_{\tau}^{\alpha}(\Theta)} \sim\left(\sum_{\mu \in \mathcal{M}}\left\|d_{\mu}(f) F_{\mu}\right\|_{p}^{\tau}\right)^{1 / \tau} .
$$

The following embedding result indicates why these spaces work in nonlinear approximation. In fact, it shows that $\dot{B}_{\tau}^{\alpha}(\Theta)$ lies on the Sobolev embedding line.

Theorem 6.1. The B-space $\dot{B}_{\tau}^{\alpha}(\Theta)$, with $p, \tau$ interrelated by (6.1), is continuously embedded in $L_{p}$, that is, each $f \in \dot{B}_{\tau}^{\alpha}(\Theta)$ can be identified as a function $f \in L_{p}$ and

$$
\|f\|_{p} \leq c\|f\|_{\dot{B}_{\tau}^{\alpha}(\Theta)}
$$

Proof. Property (a) and (c) of discrete ellipsoid covers imply that for any $x \in \mathbb{R}^{n}$ and $\theta \in \Theta_{j}(j \in \mathbb{Z})$ such that $x \in \theta$

$$
\sum_{m \leq j} \sum_{\eta \in \Theta_{m}: x \in \theta}(|\theta| /|\eta|)^{1 / p} \leq c,
$$


where $c$ is independent of $x$ and $\theta$. We now invoke Theorem 3.3 from [14], which shows that (6.6) leads to

$$
\left\|\sum_{\mu \in \mathcal{M}}\left|d_{\mu}(f) F_{\mu}(\cdot)\right|\right\|_{p} \leq c\left(\sum_{\mu \in \mathcal{M}}\left\|d_{\mu}(f) F_{\mu}\right\|_{p}^{\tau}\right)^{1 / \tau}
$$

We now consider the inhomogeneous B-space $B_{\tau}^{\alpha}:=B_{\tau \tau}^{\alpha}\left(\Theta^{+}\right)$associated with a discrete ellipsoid cover $\Theta^{+}=\cup_{j \geq 0} \Theta_{j}$ of $\mathbb{R}^{n}$. The norm in $B_{\tau}^{\alpha}$ has a representation similar to (6.4), namely,

$$
\|f\|_{B_{\tau}^{\alpha}(\Theta)} \sim\left(\sum_{\mu \in \mathcal{M}^{+}}\left\|d_{\mu}(f) F_{\mu}\right\|_{p}^{\tau}\right)^{1 / \tau}, \quad \mathcal{M}^{+}:=\cup_{j \geq-1} \mathcal{M}_{j},
$$

and an embedding result similar to Theorem 6.1 holds.

6.2. Jackson estimates for nonlinear approximation. Consider nonlinear $m$ term approximation from $\mathcal{F}:=\cup_{j \in \mathbb{Z}} \mathcal{F}_{j}=\left\{F_{\mu}: \mu \in \mathcal{M}\right\}$. We let $\mathcal{E}_{m}$ denote the nonlinear set of all functions $g$ of the form

$$
g=\sum_{\mu \in \Gamma_{m}} a_{\mu} F_{\mu},
$$

where $\Gamma_{m} \subset \mathcal{M}, \# \Gamma \leq m$, and $\Gamma$ is allowed to vary with $g$. We denote by $\sigma_{m}(f)_{p}$ the error of best $L_{p}$-approximation to $f \in L_{p}\left(\mathbb{R}^{n}\right)$ from $\mathcal{E}_{m}$ :

$$
\sigma_{m}(f)_{p}:=\inf _{g \in \mathcal{E}_{m}}\|f-g\|_{p} .
$$

Theorem 6.2 (Jackson estimate). If $f \in \dot{B}_{\tau}^{\alpha}(\Theta), \alpha>0,0<p \leq \infty$, then

$$
\sigma_{m}(f)_{p} \leq c m^{-\alpha / n}\|f\|_{\dot{B}_{\tau}^{\alpha}(\Theta)},
$$

where $c$ depends only on $\alpha, p$, and the parameters of $\Theta$.

In the case $0<p<\infty$, estimate (6.8) follows by the general Theorem 3.4 from [14], using (6.6). The proof of (6.8) in the case $p=\infty$ is more involved and can be carried out as the proof of Theorem 3.1 in [15]. It will be omitted.

The nonlinear $m$-term approximation $\sigma_{m}(f)_{p}$ from $\mathcal{F}^{+}:=\cup_{j>-1} \mathcal{F}_{j}$, where $\mathcal{F}_{-1}:=\left\{g_{\lambda}: \lambda \in \Lambda_{0}\right\}$, can be defined as above by simply replacing $\mathcal{M}$ by $\mathcal{M}^{+}$. Then the Jackson estimate (6.8) holds with $\dot{B}_{\tau}^{\alpha}(\Theta)$ replaced by $B_{\tau}^{\alpha}(\Theta)$. We omit the details.

The Jackson estimate (6.8) readily yields a direct estimate for nonlinear $m$-term approximation from $\mathcal{F}\left(\right.$ or $\mathcal{F}^{+}$) by the $K$-functional between $L_{p}$ and $\dot{B}_{\tau}^{\alpha}(\Theta)$ (or $B_{\tau}^{\alpha}(\Theta)$ ). It is a challenging open problem to prove a companion inverse estimate due to the fact that $\mathcal{F}$ is possibly redundant and nonnested.

\section{Measuring Smoothness via anisotropic B-SPaCes}

It is an ancient question in analysis, what is the smoothness of a given function and how should the smoothness be measured. In this section we show how the anisotropic B-spaces $\dot{B}_{\tau}^{\alpha}(\Theta)$ developed in the previous sections can be employed for handling this question and how it is related to nonlinear $m$-term approximation from the two-level-split bases developed here.

We consider two "simple" examples of discontinuous functions on $\mathbb{R}^{2}$, namely, $\mathbb{1}_{B(0,1)}$ the characteristic function of the unit disk $B(0,1)$ and $\mathbb{1}_{Q}$ the characteristic 
function of a square $Q \subset \mathbb{R}^{2}$. Both examples could be generalized at the expense of additional technical efforts. But in the present simple form they already suffice to reveal some interesting and perhaps surprising facts. Indeed, we shall show that each of these functions has higher order smoothness $\alpha$ in $\dot{B}_{\tau}^{\alpha}(\Theta)$ for appropriately selected ellipse cover $\Theta$ compared with its (classical) Besov space smoothness. Moreover, their smoothness via suitable covers will be seen to differ substantially.

As in the previous section, for given $0<p<\infty$ and $\alpha>0, \tau$ is defined by $1 / \tau=\alpha / 2+1 / p$.

\subsection{The anisotropic B-space smoothness of $\mathbb{1}_{B(0,1)}$.}

Theorem 7.1. There exists an anisotropic ellipsoid cover $\Theta$ of $\mathbb{R}^{2}$ such that $\mathbb{1}_{B(0,1)} \in B_{\tau}^{\alpha}(\Theta)$ for any $\alpha<4 / p$. In comparison, in the scale of Besov spaces $B_{\tau \tau}^{\alpha}$ one has $\mathbb{1}_{B(0,1)} \in B_{\tau \tau}^{\alpha}$ for $\alpha<2 / p$. Here the bounds for $\alpha$ are sharp.

Proof. We begin by constructing an appropriate continuous ellipse cover $\Theta_{\text {con }}$ of $\mathbb{R}^{2}$. For arbitrary $t \leq 0$ and $v \in \mathbb{R}^{2}$, we define the ellipse $\theta(v, t)$ by

$$
\theta(v, t):=2^{-t / 2} B(0,1)+v,
$$

that is the disk of radius $2^{-t / 2}$ centered at $v$.

Let $t>0$. For any $v=\left(v_{1}, 0\right)$ on the positive $x_{1}$-axis which obeys the condition $\left|1-v_{1}\right| \leq 2^{-t / 3}$, we define $\theta(v, t)$ as the set of all point $x \in \mathbb{R}^{2}$ such that

$$
\frac{\left(x_{1}-v_{1}\right)^{2}}{\sigma_{1}^{2}}+\frac{x_{2}^{2}}{\sigma_{2}^{2}} \leq 1
$$

where $\sigma_{1}:=\left(\left|1-v_{1}\right|+2^{-t / 2}\right) 2^{-t / 6}$ and $\sigma_{2}:=\left(\left|1-v_{1}\right|+2^{-t / 2}\right)^{-1} 2^{-5 t / 6}$.

If $v=\left(v_{1}, 0\right), v_{1} \geq 0$, satisfies $\left|1-v_{1}\right|>2^{-t / 3}$, we set $\theta(v, t):=2^{-t / 2} B(0,1)+v$.

For any point $v$ which does not lie on the positive $x_{1}$-axis we define $\theta(v, t)$ by a rotation of the ellipse $\theta(|v|, t)$ defined above about the origin which takes $(|v|, 0)$ to $v$.

We now show that the collection of ellipses $\Theta_{\text {con }}$ defined above is a continuous ellipse cover of $\mathbb{R}^{2}$ in the sense of Definition 2.4.

Fix $v=\left(v_{1}, 0\right), v_{1}>0$. Let $t, s>0$ and assume that $\left|1-v_{1}\right| \leq 2^{-(t+s) / 3}$. (All other cases are similar or trivial.) Denote by $\sigma_{1}(t)$ the $x_{1}$-semi-axis of $\theta(v, t)$. By the definition we have

$$
\frac{\sigma_{1}(t+s)}{\sigma_{1}(t)}=\frac{\left|1-v_{1}\right|+2^{-(t+s) / 2}}{\left|1-v_{1}\right|+2^{-t / 2}} \cdot 2^{-s / 6},
$$

which leads to

$$
2^{-2 s / 3} \leq \sigma_{1}(t+s) / \sigma_{1}(t) \leq 2^{-s / 6} .
$$

The case when $v$ does not lie on the positive $x_{1}$-axis reduces to the above by rotation.

Now, fix $t>0$ and let $\theta:=\theta(v, t)$ and $\theta^{\prime}:=\theta\left(v^{\prime}, t\right)$ be such that $\theta \cap \theta^{\prime} \neq \emptyset$. Assume that $|1-| v|| \leq 2^{-t / 3}$ and $|1-| v^{\prime}|| \leq 2^{-t / 3}$; the other cases are similar or trivial. Since $\Theta_{\text {con }}$ is rotation invariant, we may assume that $v=\left(v_{1}, 0\right), v_{1}>0$. Denote by $\sigma_{1}, \sigma_{2}$ the semi-axes of $\theta$ and by $\sigma_{1}^{\prime}, \sigma_{2}^{\prime}\left(\sigma_{1}^{\prime}<\sigma_{2}^{\prime}\right)$ the semi-axes of $\theta^{\prime}$. A simple rotation argument gives

$$
|| v^{\prime}|-| v|| \leq \sigma_{1}^{\prime}+\sigma_{1}=\left(|1-| v^{\prime}||+|1-| v||+2 \cdot 2^{-t / 2}\right) 2^{-t / 6} \leq 4 \cdot 2^{-t / 2}
$$


which implies

$$
\sigma_{1}^{\prime} \leq\left(|1-| v||+|| v^{\prime}|-| v||+2^{-t / 2}\right) 2^{-t / 6} \leq\left(|1-| v||+5 \cdot 2^{-t / 2}\right) 2^{-t / 6} \leq 5 \sigma_{1} .
$$

Therefore,

$$
1 / 5 \leq \sigma_{1}^{\prime} / \sigma_{1} \leq 5, \quad \text { and hence } 1 / 5 \leq \sigma_{2}^{\prime} / \sigma_{2} \leq 5 .
$$

We may assume that $t \geq 3$ (the case $t<3$ is trivial). Then $1 / 2 \leq|v|,\left|v^{\prime}\right| \leq 3 / 2$. Since $\theta \cap \theta^{\prime} \neq \emptyset$, the ellipse $\theta^{\prime}$ can be obtained by revolving $\theta\left(\left|v^{\prime}\right|, t\right)$ about the origin at an angle $\gamma$ such that

$$
|\gamma| \leq 2 \sigma_{2}+2 \sigma_{2}^{\prime} \leq 4 \cdot 2^{-t / 3}
$$

As in $\S 2$, let $A_{\theta}$ be the affine transform which maps $B(0,1)$ onto $\theta$. Then obviously $A_{\theta}(x)=M_{\theta} x+v$, where $M_{\theta}=\operatorname{diag}\left(\sigma_{1}, \sigma_{2}\right)$. The affine transform $A_{\theta^{\prime}}$ mapping $B(0,1)$ onto $\theta^{\prime}$ is of the form $A_{\theta^{\prime}}(x)=M_{\theta^{\prime}} x+v^{\prime}$, where $M_{\theta^{\prime}}$ can be represented as the product of a diagonal and a rotation matrices, namely,

$$
M_{\theta^{\prime}}=A_{\gamma} \operatorname{diag}\left(\sigma_{1}^{\prime}, \sigma_{2}^{\prime}\right), \quad A_{\gamma}:=\left(\begin{array}{cc}
\cos \gamma & -\sin \gamma \\
\sin \gamma & \cos \gamma
\end{array}\right) .
$$

It is straightforward to show that

$$
M_{\theta}^{-1} M_{\theta^{\prime}}=\left(\begin{array}{cc}
\left(\sigma_{1}^{\prime} / \sigma_{1}\right) \cos \gamma & -\left(\sigma_{2}^{\prime} / \sigma_{1}\right) \sin \gamma \\
\left(\sigma_{1}^{\prime} / \sigma_{2}\right) \sin \gamma & \left(\sigma_{2}^{\prime} / \sigma_{2}\right) \cos \gamma
\end{array}\right) .
$$

From (7.3) it follows that

$$
\left(\sigma_{2}^{\prime} / \sigma_{1}\right)|\sin \gamma| \leq 4 \quad \text { and } \quad\left(\sigma_{1}^{\prime} / \sigma_{2}\right)|\sin \gamma| \leq 4 .
$$

This and (7.2) imply that all entries of $M_{\theta}^{-1} M_{\theta^{\prime}}$ are bounded in absolute value by 5 . Hence

$$
\left\|M_{\theta}^{-1} M_{\theta^{\prime}}\right\|_{\ell_{2} \rightarrow \ell_{2}} \leq c \text { and similarly }\left\|M_{\theta^{\prime}}^{-1} M_{\theta}\right\|_{\ell_{2} \rightarrow \ell_{2}} \leq c .
$$

From this and (7.1) it follows that $\Theta_{\text {con }}$ is a continuous ellipse cover of $\mathbb{R}^{2}$.

In applying Lemma 2.9 to $\Theta_{\text {con }}$ we conclude that there is a discrete ellipse cover $\Theta$ of $\mathbb{R}^{2}$ such that each ellipse $\theta \in \Theta$ is a dilated version of an ellipse from $\Theta_{\text {con }}$ by a factor $\rho_{\theta}$ obeying $\left(a_{7}+1\right) / 2 \leq \rho_{\theta} \leq 1$. Also, for any $\theta \in \Theta_{m}$ we have $a_{1} 2^{-m} \leq|\theta| \leq a_{2} 2^{-m}$, where $a_{1}, a_{2}$ are positive constants.

It remains to show that $\mathbb{1}_{B(0,1)} \in \dot{B}_{\tau}^{\alpha}(\theta)$ for all $\alpha<4 / p$. Denote by $\Theta_{m}^{\prime}$ the set of all ellipses from $\Theta$ which intersect the portion $S^{1}$ of $B(0,1)$ that belongs to the positive quadrant. We need to estimate $\# \Theta_{m}^{\prime}$. By condition (c) on discrete ellipsoid covers, only $N_{1}$ ellipses from $\Theta_{m}$ may intersect at a time. This and the construction of $\Theta_{\text {con }}$ and $\Theta$ yield

$$
\# \Theta_{m}^{\prime} \leq c 2^{m}\left|\bigcup_{\theta \in \Theta_{m}^{\prime}} \theta\right| \leq c 2^{m} 2^{-2 m / 3}=c 2^{m / 3}, \quad m>0 .
$$

Evidently, $\# \Theta_{m}^{\prime} \leq c$ if $m \leq 0$. 
We use these to estimate $\|f\|_{\dot{B}_{\tau}^{\alpha}(\Theta)}$, where $f:=\mathbb{1}_{B(0,1)}$. Evidently, $\omega_{k}(f, \theta)_{\tau}=0$ if $\theta \in \Theta_{m} \backslash \Theta_{m}^{\prime}$ and hence

$$
\begin{aligned}
\|f\|_{\dot{B}_{\tau}^{\alpha}(\Theta)}^{\tau} & =\sum_{m \in \mathbb{Z}} \sum_{\theta \in \Theta_{m}^{\prime}}|\theta|^{-\alpha \tau / 2} \omega_{k}(f, \theta)_{\tau}^{\tau} \\
& \leq c \sum_{m=-\infty}^{0} 2^{m \alpha \tau / 2}+c \sum_{m=1}^{\infty}\left(\# \Theta_{m}^{\prime}\right) 2^{-m(1-\alpha \tau / 2)} \\
& \leq c+c \sum_{m=1}^{\infty} 2^{-m(2 / 3-\alpha \tau / 2)} \leq c<\infty
\end{aligned}
$$

where we used that $2 / 3-\alpha \tau / 3=(2 \tau / 3)(1 / p-\alpha / 4)>0$, since $\alpha<4 / p$. Consequently, $f \in \dot{B}_{\tau}^{\alpha}(\Theta)$. Here the bound $4 / p$ for $\alpha$ is sharp since $S^{1}$ cannot be covered by $\leq c 2^{m / 3}$ ellipsoids of area $2^{-m}$ whenever $c>0$ is sufficiently small.

It is well known that $\mathbb{1}_{B(0,1)}$ belongs to the Besov space $B_{\tau \tau}^{\alpha}$ for $\alpha<2 / p$ and this bound for $\alpha$ is sharp.

As in $\S 6.2$ denote by $\sigma_{m}(f)_{p}$ the best $m$-term approximation of $f$ from $\mathcal{F}$ in $L_{p}$. The following estimate is immediate from Theorem 7.1 and Theorem 6.2.

Corollary 7.2. Let $0<p<\infty$. Then

$$
\sigma_{m}\left(\mathbb{1}_{B(0,1)}\right)_{p} \leq \mathrm{cm}^{-\gamma} \text { for all } \gamma<2 / p
$$

At the same time, if $\sigma_{m}^{W}(f)_{p}$ denotes the best $m$-term approximation of $f$ in $L_{p}$ $(p \geq 1)$ from any reasonable wavelet basis, then

$$
\sigma_{m}^{W}\left(\mathbb{1}_{B(0,1)}\right)_{p} \leq c m^{-\gamma} \text { for all } \gamma<1 / p .
$$

Here both estimate are sharp.

7.2. The anisotropic B-space smoothness of $\mathbb{1}_{Q}$. We assume the notation from $\S 7.1$.

Theorem 7.3. For any square $Q$ in $\mathbb{R}^{2}$ and any $\alpha>0$ there exists an anisotropic ellipsoid cover $\Theta$ of $\mathbb{R}^{2}$ such that $\mathbb{1}_{Q} \in B_{\tau}^{\alpha}(\Theta)$, while in the scale of Besov spaces $B_{\tau \tau}^{\alpha}$ one has only $\mathbb{1}_{Q} \in B_{\tau \tau}^{\alpha}$ for $\alpha<2 / p$ and this bound for $\alpha$ is sharp.

Proof. Without loss of generality we may assume that $Q=[-1,1] \times[0,2]$. It is convenient to construct first an appropriate continuous ellipse cover and then get the desired discrete ellipse cover upon employing Lemma 2.9.

Our main step is to effectively define the ellipses $\theta(v, t)$ of our continuous cover $\Theta_{\text {con }}$ with centers $v$ from the triangle $\triangle_{0}:=[(0,0),(1,0),(0,1)]$ and $t>0$. Then we use symmetry about the $x_{2}$-axis to define $\theta(v, t)$ for $v$ in the triangle

$$
[(-1,0),(0,0),(0,1)] \text {. }
$$

We next apply symmetry about the $x_{1}$-axis to define the ellipses $\theta(v, t)$ for $v$ in the triangle $[(-1,0),(0,-1),(1,0)]$. Again by symmetry about the line $x_{2}=-x_{1}+1$ we define $\theta(v, t)$ on the square $[(1,0),(2,1),(1,2),(0,1)]$. Symmetry about the line $x_{2}=x_{1}+1$ enables us to define $\theta(v, t)$ for $v$ in the rectangle

$$
[(-1,0),(1,2),(0,3)),(-2,1))] \text {. }
$$

In this way the ellipses $\theta(v, t)$ would be defined with centers $v$ from the square

$$
S:=[(0,-1),(2,1),(0,3),(-2,1)] .
$$


Finally, we define the ellipses $\theta(v, t)$ with centers $v \in \mathbb{R}^{2} \backslash S$ by

$$
\theta(v, t):=2^{-t / 2} B(0,1)+v
$$

In going further, for $t \leq 0$ we define $\theta(v, t)$ for all centers $v \in \mathbb{R}^{2}$ by $\theta(v, t):=$ $2^{-t / 2} B(0,1)+v$.

It remains to define the ellipses $\theta(v, t)$ with centers $v \in \triangle_{0}$ and $t>0$. We begin by introducing a parameter $\delta$ determined by the condition

$$
0<\delta<\min \left\{\frac{1}{2}, \frac{4}{2+p \alpha}\right\} \text {. }
$$

For every $v \in \triangle_{0}, v=\left(v_{1}, v_{2}\right)$, set $u:=u_{v}:=1-v_{1}-v_{2}$ and define $\theta(v, t)$ as the set of all $x \in \mathbb{R}^{2}$ such that

$$
\frac{\left(x_{1}-v_{1}\right)^{2}}{\sigma_{1}^{2}}+\frac{\left(x_{2}-v_{2}\right)^{2}}{\sigma_{2}^{2}} \leq 1,
$$

where $\sigma_{1}:=\left(u_{v} 2^{t / 2}+1\right)^{1-\delta} 2^{-t / 2}$ and $\sigma_{2}:=\left(u_{v} 2^{t / 2}+1\right)^{\delta-1} 2^{-t / 2}$. Evidently, $|\theta(v, t)|=\pi \sigma_{1} \sigma_{2}=\pi 2^{-t}$. Notice also that $\theta(v, t):=2^{-t / 2} B(0,1)+v$ if $u_{v}=0$ which is the same as $v \in[(1,0),(0,1)]$.

We next show that the collection of ellipses $\Theta_{\text {con }}$ defined above is a continuous ellipse cover of $\mathbb{R}^{2}$ with parameters depending only on $\delta$.

Fix $v \in \triangle_{0}$ and for any $t \in \mathbb{R}$ denote by $\sigma_{1}(t)$ and $\sigma_{2}(t)$ the semi-axes of $\theta(v, t)$. Then from above we have for $t, s>0$

$$
\frac{\sigma_{1}(t+s)}{\sigma_{1}(t)}=\left(\frac{u_{v}+2^{-(t+s) / 2}}{u_{v}+2^{-t / 2}}\right)^{1-\delta} 2^{-\delta s / 2},
$$

which readily implies

$$
2^{-s / 2} \leq \sigma_{1}(t+s) / \sigma_{1}(t) \leq 2^{-\delta s / 2}
$$

Now, fix $t>0$ and let $\theta(v, t) \cap \theta\left(v^{\prime}, t\right) \neq \emptyset, v, v^{\prime} \in \triangle_{0}$. Assume $u_{v^{\prime}}>u_{v}$. Clearly,

$$
\begin{aligned}
u_{v^{\prime}} & \leq u_{v}+\sigma_{1}(v)+\sigma_{1}\left(v^{\prime}\right) \\
& \leq u_{v}+\left(u_{v}+2^{-t / 2}\right)^{1-\delta} 2^{-\delta t / 2}+\left(u_{v^{\prime}}+2^{-t / 2}\right)^{1-\delta} 2^{-\delta t / 2} \\
& \leq u_{v}+2 u_{v}^{1-\delta} 2^{-\delta t / 2}+2 \cdot 2^{-t / 2}+2 u_{v^{\prime}}^{1-\delta} 2^{-\delta t / 2}+2 \cdot 2^{-t / 2} .
\end{aligned}
$$

If $u_{v^{\prime}} \geq 4^{1 / \delta} 2^{-t / 2}$, this leads to

$$
u_{v^{\prime}} \leq 8\left(u_{v}+u_{v}^{1-\delta} 2^{-\delta t / 2}+2^{-t / 2}\right) \leq 16\left(u_{v}+2^{-t / 2}\right)
$$

which yields

$$
\sigma_{1}\left(v^{\prime}\right) / \sigma_{1}(v) \leq c, \quad c=c(\delta)
$$

If $u_{v^{\prime}}<4^{1 / \delta} 2^{-t / 2}$, the same estimate follows immediately with a different constant c.

Estimates (7.5)-(7.6) readily imply that $\Theta_{\text {con }}$ is a continuous ellipse cover of $\mathbb{R}^{2}$ in the sense of Definition 2.4.

By Lemma 2.9 the above cover $\Theta_{\text {con }}$ induces a discrete ellipse cover, say, $\Theta$ of $\mathbb{R}^{2}$, where every $\theta \in \Theta$ is obtained by dilating an ellipse from $\Theta_{\text {con }}$ (with the same center) by a factor $\rho_{\theta}$ obeying $\left(a_{7}+1\right) / 2 \leq \rho_{\theta} \leq 1$. Also, for any $\theta \in \Theta_{m}$ we have $a_{1} 2^{-m} \leq|\theta| \leq a_{2} 2^{-m}$ for some positive constants $a_{1}, a_{2}$.

Our next task is to show that the function $\mathbb{1}_{Q}$, where $Q:=[-1,1] \times[0,2]$, belongs to the B-space $\dot{B}_{\tau}^{\alpha}(\Theta)$. To this end we need an upper bound for the number of all ellipses from $\Theta_{m}$ which intersect the boundary of $[-1,1] \times[0,2]$. Denote this set 
by $\Theta_{m}^{\prime}$. By condition (c) on discrete covers and the construction of $\Theta_{\text {con }}$ and $\Theta$ it follows that for $m>0$

$$
\begin{aligned}
\# \Theta_{m}^{\prime} & \leq c 2^{m}\left|\bigcup_{\theta \in \Theta_{m}^{\prime}} \theta\right| \leq c 2^{m} \int_{0}^{1} \sigma_{2}\left(\theta\left(v_{1}, 0\right), 2^{-m}\right) d v_{1} \\
& \leq c 2^{m / 2} \int_{0}^{1}\left(\left(1-v_{1}\right) 2^{m / 2}+1\right)^{\delta-1} d v_{1}=c 2^{\delta m / 2} .
\end{aligned}
$$

Evidently $\# \Theta_{m} \leq c$ if $m \leq 0$.

We are now prepared to estimate $\|f\|_{\dot{B}_{\tau}^{\alpha}(\Theta)}$, where $f:=\mathbb{1}_{Q}$. Evidently, $\omega_{k}(f, \theta)_{\tau}=$ 0 if $\theta \in \Theta_{m} \backslash \Theta_{m}^{\prime}$. From this and the above estimates of $\# \Theta_{m}^{\prime}$ we get

$$
\begin{aligned}
\|f\|_{\dot{B}_{\tau}^{\alpha}(\Theta)}^{\tau} & =\sum_{m \in \mathbb{Z}} \sum_{\theta \in \Theta_{m}^{\prime}}|\theta|^{-\alpha \tau / 2} \omega_{k}(f, \theta)_{\tau}^{\tau} \\
& \leq c \sum_{m=-\infty}^{0} 2^{m \alpha \tau / 2}+c \sum_{m=1}^{\infty}\left(\# \Theta_{m}^{\prime}\right) 2^{-m(1-\alpha \tau / 2)} \\
& \leq c+c \sum_{m=1}^{\infty} 2^{-m(1-\alpha \tau / 2-\delta / 2)} \leq c<\infty .
\end{aligned}
$$

Here we used that $1-\alpha \tau / 2-\delta / 2=\tau(1 / \tau-\alpha / 2-\delta / 2 \tau)=\tau(1 / p-\delta / 2 \tau)>0$ by (7.4). Thus $f \in \dot{B}_{\tau}^{\alpha}(\Theta)$.

It is standard and well known that $\mathbb{1}_{Q}$ belongs to the Besov space $B_{\tau \tau}^{\alpha}$ if $\alpha<2 / p$ and this bound for $\alpha$ is sharp.

As above Theorem 7.1 and Theorem 6.2 lead to the following approximation result.

Corollary 7.4. For any $\alpha>0$ there exists a discrete ellipse cover of $\mathbb{R}^{2}$ such that for all $0<p<\infty$

$$
\sigma_{m}\left(\mathbb{1}_{Q}\right)_{p} \leq c m^{-\alpha}
$$

In comparison, for wavelet $m$-term approximation of $\mathbb{1}_{Q}$ in $L_{p}(p \geq 1)$ one has only

$$
\sigma_{m}^{W}\left(\mathbb{1}_{Q}\right)_{p} \leq c m^{-\gamma} \quad \text { for all } \quad \gamma<1 / p,
$$

and this estimate is sharp.

7.3. Discussion. One of the basic goals in this paper is to advance regularity notions that better respect anisotropic features of functions. The key idea is to measure the smoothness of functions adaptively by means of anisotropic B-spaces, and in particular by the B-spaces of nonlinear approximation $\dot{B}_{\tau}^{\alpha}(\Theta)$, by allowing the underlying ellipsoid covers to adapt to the given function. We have considered two model problems which clearly shed some light on this idea. First, as was shown in $\S 7.1-\S 7.2$ for appropriate ellipse covers, the B-space smoothness of the characteristic functions of the unit ball and any square in $\mathbb{R}^{2}$ is higher than their Besov space smoothness. It is rather obvious that by using adaptive dilations the anisotropic B-spaces are better able to resolve the singularities along smooth or piecewise smooth curves. As a result the two-level-split decompositions of these functions are sparser than their wavelet decompositions, which leads to better rates of nonlinear $m$-term approximation. Second, it might surprise that characteristic functions of polygonal domains have infinite smoothness while those of domains 
with smooth boundaries have limited regularity. Roughly speaking the positive curvature in the latter case limits the extent to which ellipses are allowed to stretch. On the other hand, the covers that would yield higher and higher smoothness in the polygonal case have to become less and less constrained, which means that the parameters in $\mathbf{p}(\Theta)$ are subjected to more and more generous bounds. Keeping these parameters within a compact set would limit the regularity that could be described in this way. Thus the idea of describing smoothness by maximizing $\alpha$ over covers $\Theta$ has to be treated with care. This maximum could be determined by a saturated smoothness as in the first example. Or such a maximum would be achieved only when constraining the parameter sets of the covers to some a-priori chosen compact set which is not an intrinsic property of the underlying function. Finally, it is a challenging open problem to devise a scheme which for a given function $f$ finds an optimal (or near optimal) ellipsoid cover $\Theta$ such that $f$ exhibits the highest order $\alpha$ of smoothness in $\dot{B}_{\tau}^{\alpha}(\Theta)$ in the above sense.

\section{APPENDIX}

Proof of Theorem 5.8. Evidently, $\|f\|_{\dot{B}_{p q}^{\alpha}(\Theta)}^{A} \leq\|f\|_{\dot{B}_{p q}^{\alpha}(\Theta)}^{T}$ and we have already shown that $\|f\|_{\dot{B}_{p q}^{\alpha}(\Theta)}^{T} \leq c\|f\|_{\dot{B}_{p q}^{\alpha}(\Theta)}$.

It remains to prove that

$$
\|f\|_{\dot{B}_{p q}^{\alpha}(\Theta)} \leq c\|f\|_{\dot{B}_{p q}^{\alpha}(\Theta)}^{A} .
$$

We only consider the least favorable case when $1<p<q<\infty$. Let $f=$ $\sum_{\mu \in \mathcal{M}} a_{\mu} F_{\mu}$. For any ellipsoid $\sigma \in \Theta_{j}$ we have

$$
\begin{aligned}
& \omega_{k}(f, \sigma)_{p} \leq \omega_{k}\left(\sum_{\mu: \ell\left(\eta_{\mu}\right)<j} a_{\mu} F_{\mu}, \sigma\right)_{p}+c\left\|\sum_{\mu: \ell\left(\eta_{\mu}\right) \geq j} a_{\mu} F_{\mu}\right\|_{p} \\
& (8.2) \quad \leq c \sum_{\nu=1}^{\infty}\left(\sum_{\mu: \eta_{\mu} \in \Theta_{j-\nu}}\left|a_{\mu}\right|^{p} \omega_{k}\left(F_{\mu}, \sigma\right)_{p}^{p}\right)^{1 / p}+c \sum_{\nu=0}^{\infty}\left(\sum_{\mu: \eta_{\mu} \in \Theta_{j+\nu}}\left\|a_{\mu} F_{\mu}\right\|_{p}^{p}\right)^{1 / p} .
\end{aligned}
$$

Here $\ell(\eta)$ stands for the level of $\eta$ in $\Theta$.

We next estimate $\omega_{k}\left(F_{\mu}, \sigma\right)_{p}$ for any $\mu \in \mathcal{M}$ such that $\eta_{\mu} \in \Theta_{j-\nu}, \nu \geq 1$. Denote briefly $\eta:=\eta_{\mu}, \theta:=\theta_{\mu}, \beta:=\beta_{\mu}$, and $F:=F_{\mu}$. Also let $\sigma^{*}:=A_{\eta}^{-1} \sigma=$ $A_{\eta}^{-1} A_{\sigma} B^{*}, \theta^{*}:=A_{\eta}^{-1} \theta$, and $F^{*}:=F \circ A_{\eta}$. Recall that $F=P_{\eta, \beta} \varphi_{\eta} \varphi_{\theta}$ with $P_{\eta, \beta}:=|\eta|^{-1 / 2} P_{\beta} \circ A_{\eta}^{-1}$. Hence $F^{*}=|\eta|^{-1 / 2} P_{\beta}\left(\varphi_{\eta} \circ A_{\eta}\right)\left(\varphi_{\theta} \circ A_{\eta}\right)$.

For any $h \in \mathbb{R}^{n}$ we have with $h^{*}:=A_{\eta}^{-1} h$

$$
\begin{aligned}
\left\|\Delta_{h}^{k} F\right\|_{L_{p}(\sigma)}^{p} & =\left|\operatorname{det} M_{\eta}\right|\left\|\Delta_{h^{*}}^{k} F^{*}\right\|_{L_{p}\left(\sigma^{*}\right)}^{p} \leq c|\eta|\left|h^{*}\right|^{k p}\left\|\left(\frac{\partial}{\partial h}\right)^{k} F^{*}\right\|_{\infty}^{p}\left|\sigma^{*}\right| \\
& \leq c\left|\eta\left\|\sigma^{*} \mid\left(\operatorname{diam} \sigma^{*}\right)^{k p}\right\| F^{*} \|_{\infty}^{p} .\right.
\end{aligned}
$$

Here we assumed that $k\left|h^{*}\right| \leq \operatorname{diam} \sigma^{*}$, since otherwise $\Delta_{h^{*}}^{k} F^{*}(x) \equiv 0$. We also used that $\left\|\left(\frac{\partial}{\partial h}\right)^{k} F^{*}\right\|_{\infty} \leq c$ with $c$ a constant depending only on $\mathbf{p}(\Theta), k$, and $L$.

Note that $\left\|F^{*}\right\|_{\infty}=\|F\|_{\infty}$ and hence $\|F\|_{p}^{p} \sim|\eta|\left\|F^{*}\right\|_{\infty}^{p}$. Also, we have

$$
\left|\sigma^{*}\right|=\left|\operatorname{det}\left(M_{\eta}^{-1} M_{\sigma}\right)\right|=\left|\operatorname{det} M_{\eta}^{-1}\right|\left|\operatorname{det} M_{\sigma}\right|=|\eta|^{-1}|\sigma|
$$

and $\operatorname{diam} \sigma^{*}=2\left\|M_{\eta}^{-1} M_{\sigma}\right\|_{\ell_{2} \rightarrow \ell_{2}} \leq 2 a_{5} 2^{-a_{6} \nu}$ using property (b) of $\Theta$. We use the above observations in (8.3) to obtain

$$
\omega_{k}\left(F_{\mu}, \sigma\right)_{p}^{p} \leq c|\sigma|\left|\eta_{\mu}\right|^{-1} 2^{-a_{6} \nu k p}\left\|F_{\mu}\right\|_{p}^{p} .
$$


Combining this with (8.2), we get

$$
\begin{aligned}
|\sigma|^{-\alpha p / n} & \omega_{k}(f, \sigma)_{p}^{p} \\
& \leq c\left[\sum_{\nu=1}^{\infty}\left(\sum_{\mu: \eta_{\mu} \in \Theta_{j-\nu}, \eta_{\mu} \cap \sigma \neq \emptyset}\left(\frac{\left|\eta_{\mu}\right|}{|\sigma|}\right)^{\alpha p / n-1} 2^{-a_{6} \nu k p}\left|\eta_{\mu}\right|^{-\alpha p / n}\left\|a_{\mu} F_{\mu}\right\|_{p}^{p}\right)^{1 / p}\right]^{p} \\
& +c\left[\sum_{\nu=0}^{\infty}\left(\sum_{\mu: \eta_{\mu} \in \Theta_{j+\nu}, \eta_{\mu} \cap \sigma \neq \emptyset}\left(\frac{\left|\eta_{\mu}\right|}{|\sigma|}\right)^{\alpha p / n}\left|\eta_{\mu}\right|^{-\alpha p / n}\left\|a_{\mu} F_{\mu}\right\|_{p}^{p}\right)^{1 / p}\right]^{p} \\
& \leq c\left[\sum_{\nu=1}^{\infty}\left(\sum_{\mu: \eta_{\mu} \in \Theta_{j-\nu}, \eta_{\mu} \cap \sigma \neq \emptyset} 2^{-\nu p\left(-a_{0} \alpha / n+a_{6} k\right)-a_{0} \nu} U_{\mu}\right)^{1 / p}\right]^{p} \\
& +c\left[\sum_{\nu=0}^{\infty}\left(\sum_{\mu: \eta_{\mu} \in \Theta_{j+\nu}, \eta_{\mu} \cap \sigma \neq \emptyset} 2^{-a_{0} \nu \alpha p / n} U_{\mu}\right)^{1 / p}\right]^{p},
\end{aligned}
$$

where $U_{\mu}:=\left|\eta_{\mu}\right|^{-\alpha p / n}\left\|a_{\mu} F_{\mu}\right\|_{p}^{p}$.

Denote briefly $\varepsilon:=-a_{0} \alpha / n+a_{6} k$ and $\delta:=a_{0} \alpha / n>0$. Notice that $\varepsilon>0$ since $k>\frac{a_{0}}{a_{6}} \cdot \frac{\alpha}{n}$ by (5.1). We use the above in the definition of $\|f\|_{\dot{B}_{p q}^{\alpha}(\Theta)}$ to obtain

$$
\begin{aligned}
\left(\|f\|_{\dot{B}_{p q}^{\alpha}}\right)^{q} & \leq c \sum_{j \in \mathbb{Z}}\left\{\sum_{\sigma \in \Theta_{j}}\left[\sum_{\nu=1}^{\infty} 2^{-\varepsilon \nu-a_{0} \nu / p}\left(\sum_{\mu: \eta_{\mu} \in \Theta_{j-\nu}, \eta_{\mu} \cap \sigma \neq \emptyset} U_{\mu}\right)^{1 / p}\right]^{p}\right\}^{q / p} \\
& +c \sum_{j \in \mathbb{Z}}\left\{\sum_{\sigma \in \Theta_{j}}\left[\sum_{\nu=0}^{\infty} 2^{-\delta \nu}\left(\sum_{\mu: \eta_{\mu} \in \Theta_{j+\nu}, \eta_{\mu} \cap \sigma \neq \emptyset} U_{\mu}\right)^{1 / p}\right]^{p}\right\}^{q / p} \\
& =: \Sigma_{1}+\Sigma_{2} .
\end{aligned}
$$

To estimate $\Sigma_{1}$ we apply Hölder's inequality and get

$$
\begin{aligned}
\Sigma_{1} & \leq c \sum_{j \in \mathbb{Z}}\left[\sum_{\sigma \in \Theta_{j}}\left(\sum_{\nu=1}^{\infty} 2^{-\varepsilon \nu p / 2-a_{0} \nu} \sum_{\mu: \eta_{\mu} \in \Theta_{j-\nu}, \eta_{\mu} \cap \sigma \neq \emptyset} U_{\mu}\right)\left(\sum_{\nu=1}^{\infty} 2^{-\varepsilon \nu p^{\prime} / 2}\right)^{p / p^{\prime}}\right]^{q / p} \\
& \leq c \sum_{j \in \mathbb{Z}}\left(\sum_{\sigma \in \Theta_{j}} \sum_{\nu=1}^{\infty} 2^{-\varepsilon \nu p / 2-a_{0} \nu} \sum_{\mu: \eta_{\mu} \in \Theta_{j-\nu}, \eta_{\mu} \cap \sigma \neq \emptyset} U_{\mu}\right)^{q / p}\left(1 / p+1 / p^{\prime}=1\right) .
\end{aligned}
$$

In going further we switch the order of summation and then apply again Hölder's inequality to obtain

$$
\begin{aligned}
\Sigma_{1} & \leq c \sum_{j \in \mathbb{Z}}\left[\sum_{\nu=1}^{\infty} 2^{-\varepsilon \nu p / 2-a_{0} \nu}\left(\sum_{\mu: \eta_{\mu} \in \Theta_{j-\nu}} U_{\mu}\right) \#\left\{\sigma \in \Theta_{j}: \sigma \cap \eta_{\mu} \neq \emptyset\right\}\right]^{q / p} \\
& \leq c \sum_{j \in \mathbb{Z}}\left[\sum_{\nu=1}^{\infty} 2^{-\varepsilon \nu p / 2} \sum_{\mu: \eta_{\mu} \in \Theta_{j-\nu}} U_{\mu}\right]^{q / p} \\
& \leq c \sum_{j \in \mathbb{Z}}\left[\sum_{\nu=1}^{\infty} 2^{-\varepsilon \nu q / 4}\left(\sum_{\mu: \eta_{\mu} \in \Theta_{j-\nu}} U_{\mu}\right)^{q / p}\right]\left[\sum_{\nu=1}^{\infty} 2^{-\varepsilon \nu p r^{\prime} / 4}\right]^{q / p r^{\prime}} \quad(r:=q / p>1),
\end{aligned}
$$

where we used that $\#\left\{\sigma \in \Theta_{j}: \sigma \cap \eta_{\mu} \neq \emptyset\right\} \leq c 2^{a_{0} \nu}$, which is a consequence of properties (a) and (c) of $\Theta$. 
Finally, we apply the substitution $l:=j-\nu$ and switch the order of summation above to obtain

$$
\begin{aligned}
\Sigma_{1} & \leq c \sum_{j \in \mathbb{Z}} \sum_{l=-\infty}^{j-1} 2^{-\varepsilon(j-l) q / 4}\left(\sum_{\mu: \eta_{\mu} \in \Theta_{l}} U_{\mu}\right)^{q / p} \\
& \leq c \sum_{l \in \mathbb{Z}}\left(\sum_{\mu: \eta_{\mu} \in \Theta_{l}} U_{\mu}\right)^{q / p} \sum_{j=l+1}^{\infty} 2^{-\varepsilon(j-l) q / 4} \leq c \sum_{l \in \mathbb{Z}}\left(\sum_{\mu: \eta_{\mu} \in \Theta_{l}} U_{\mu}\right)^{q / p} \\
& \leq\left(\|f\|_{\dot{B}_{p q}^{\alpha}}^{A}\right)^{q} .
\end{aligned}
$$

We estimate $\Sigma_{2}$ in a similar fashion. Applying Hölder's inequality we get

$$
\begin{aligned}
\Sigma_{2} & \leq c \sum_{j \in \mathbb{Z}}\left[\sum_{\sigma \in \Theta_{j}}\left(\sum_{\nu=0}^{\infty} 2^{-\delta \nu p / 2} \sum_{\mu: \eta_{\mu} \in \Theta_{j+\nu}, \eta_{\mu} \cap \sigma \neq \emptyset} U_{\mu}\right)\left(\sum_{\nu=1}^{\infty} 2^{-\delta \nu p^{\prime} / 2}\right)^{p / p^{\prime}}\right]^{q / p} \\
& \leq c \sum_{j \in \mathbb{Z}}\left(\sum_{\sigma \in \Theta_{j}} \sum_{\nu=0}^{\infty} 2^{-\delta \nu p / 2} \sum_{\mu: \eta_{\mu} \in \Theta_{j+\nu}, \eta_{\mu} \cap \sigma \neq \emptyset} U_{\mu}\right)^{q / p} .
\end{aligned}
$$

Switching the order of summation and then applying Hölder's inequality, we obtain

$$
\begin{aligned}
\Sigma_{2} & \leq c \sum_{j \in \mathbb{Z}}\left[\sum_{\nu=0}^{\infty} 2^{-\delta \nu p / 2} \sum_{\mu: \eta_{\mu} \in \Theta_{j+\nu}} U_{\mu}\right]^{q / p} \quad(r:=q / p>1) \\
& \leq c \sum_{j \in \mathbb{Z}}\left[\sum_{\nu=0}^{\infty} 2^{-\delta \nu q / 4}\left(\sum_{\mu: \eta_{\mu} \in \Theta_{j+\nu}} U_{\mu}\right)^{q / p}\right]\left[\sum_{\nu=0}^{\infty} 2^{-\delta \nu p r^{\prime} / 4}\right]^{q / p r^{\prime}} \\
& \leq c \sum_{j \in \mathbb{Z}} \sum_{l=j}^{\infty} 2^{-\delta(l-j) q / 4}\left(\sum_{\mu: \eta_{\mu} \in \Theta_{l}} U_{\mu}\right)^{q / p} \\
& \leq c \sum_{l \in \mathbb{Z}}\left(\sum_{\mu: \eta_{\mu} \in \Theta_{l}} U_{\mu}\right)^{q / p} \sum_{j=-\infty}^{l} 2^{-\delta(l-j) q / 4} \leq c \sum_{l \in \mathbb{Z}}\left(\sum_{\mu: \eta_{\mu} \in \Theta_{l}} U_{\mu}\right)^{q / p} \\
& \leq\left(\|f\|_{\dot{B}_{p q}^{\alpha}}^{A}\right)^{q} .
\end{aligned}
$$

This completes the proof of (8.1).

\section{REFERENCES}

[1] M. Bownik, Anisotrpopic Hardy spaces and wavelets, Mem. Amer. Math. Soc. 164 (2003), No 781.

[2] M. Bownik, Atomic and moleculiar decompositions of anosotrpopic Besov spaces, preprint.

[3] M. Bownik, K.-P. Ho, Atomic and moleculiar decompositions of anosotrpopic Triebel-Lizorkin spaces, Trans. Amer. Math. Soc. 358 (2006), 1469-1510.

[4] A. Calderón, A. Torchinsky, Parabolic maximal functions associated with a distribution, Adv. in Math. 16 (1975), 1-64.

[5] A. Calderón, A. Torchinsky, Parabolic maximal functions associated with a distribution II, Adv. in Math. 24 (1977), 101-171.

[6] R. Coifman, G. Weiss, Analyse harmonique non-comutative sur certains espaces homogenes, Lecture Notes in Math. 242, Springer-Verlag, 1971.

[7] R. Coifman, G. Weiss, Extensions of Hardy spaces and their use in analysis, Bull. Amer. Math. Soc. 83 (1977), 569-645.

[8] W. Dahmen, P. Petrushev, "Push-the-Error" algorithm for nonlinear n-term approximation, Constr. Approx. 23 (2006), 261-304. 
[9] W. Dahmen, S. Dekel, and P. Petrushev, Multilevel Preconditioning for Partition of Unity Methods - Some Analytic Concepts, preprint.

[10] O. Davydov, P. Petrushev, Nonlinear approximation from differentiable piecewise polynomials, SIAM J. Math. Anal. 35 (2003), 708-758.

[11] S. Dekel, D. Leviatan, and M. Sharir, On bivariate smoothness spaces associated with nonlinear approximation, Constr. Approx. 20 (2004), 625-646.

[12] G. Folland, E. Stein, Hardy spaces on homogeneous groups, Princeten University Press, N. J. 1982.

[13] Y. Han, E. Sawyer, Littlewood-Paley theory on spaces of homogeneous type and classical function spaces, Mem. Amer. Math. Soc. 530 (1994).

[14] B. Karaivanov, P. Petrushev, Nonlinear piecewise polynomial approximation beyond Besov spaces, Appl. Comput. Harmon. Anal. 15 (2003), 177-223.

[15] B. Karaivanov, P. Petrushev, and R.C. Sharpley, Algorithms for nonlinear piecewise polynomial approximation, Trans. Amer. Math. Soc., 355 (2003), 2585-2631.

[16] J. Peetre, New thoughts on Besov spaces, Duke Univ. Math. Series, Duke Univ. Durham, 1976.

[17] P. Petrushev, Anisotropic spaces and nonlinear n-term spline approximation, Approximation Theory XI: Gatlinburg 2004, 363-394, Mod. Methods Math., Nashboro Press, Brentwood, TN, 2005.

[18] H. Triebel, Theory of Function Spaces, Monographs in Math. vol. 78, Birkhäuser, 1983.

[19] H. Triebel, Theory of Function Spaces II, Monographs in Math. vol. 84, Birkhäuser, Basel, 1992

Institut für Geometrie und Praktische Mathematik, RWTH Aachen, Templergraben 55, 52056 Aachen, Germany

E-mail address: dahmen@igpm.rwth-aachen.de

Ge Healthcare, 6 Hamasger St., Or-Yehuda 60408, Israel

E-mail address: shai.dekel@ge.com

Department of Mathematics, University of South Carolina, Columbia, SC 29208, USA

E-mail address: pencho@math.sc.edu 